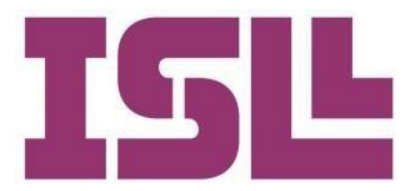

Número 11

Junio de 2019

\title{
Estrategias de aplicación digital en la comprensión de textos narrativos
}

\author{
Pedro Félix Novoa Castillo \\ Universidad Nacional Mayor de San Marcos / \\ Universidad César Vallejo \\ https://orcid.org/0000-0003-2186-7458
}

Pág. 37 a la 55

\section{Palabras clave:}

Comprensión de textos narrativos; Aprendizaje electrónico; Estrategias de lectura; Lectura digital.
La baja comprensión lectora en el ámbito universitario requiere de una urgente alternativa de solución desde lo didáctico. Por ello, las estrategias de aplicación digital son una interesante alternativa de solución. El propósito de esta investigación fue determinar la influencia que tiene un conjunto de aplicativos y programas empleados como estrategia didáctica en el proceso de lectura y comprensión de textos narrativos (en su dimensión textual, inferencial y criterial). Debido al método hipotético deductivo de la investigación se planteó como hipótesis de trabajo que la estrategia digital mejora la comprensión de textos narrativos, tanto en su dimensión textual, inferencial y criterial. Para comprobarlo, se aplicó un pre y dos pos test (adaptaciones PISA, 2015) a una muestra de 310 participantes (control: 160 y experimental: 150).
Recibido: 03-03-2019

Aceptado: 25-04-2019
Los resultados reflejaron diferencias significativas en la comprensión, tanto para su dimensión textual y criterial, mas no se comprobó significatividad en la dimensión inferencial (probablemente por el usual apresuramiento en las deducciones, situación muy común en la juventud de ahora). Por todo ello, se concluyó que las Estrategias de aplicación digital mejoran significativamente la comprensión de textos narrativos, pero que aún en el aspecto inferencial faltaría ajustar, quizá con la incorporación de una actividad didáctica más reflexiva. 


\section{Introducción}

En la presente investigación se buscó comprobar la influencia de una estrategia digital en la comprensión de textos narrativos por estudiantes universitarios. Debido a ello, se enfocó en proponer actividades que tendrían que realizarse en una o dos clases como máximo para someter a prueba intensa la relevancia de los recursos, programas y aplicativos virtuales en el logro de la comprensión tanto literal, inferencial y criterial. Las lecturas empleadas fueron dos cuentos de Jorge Luis Borges: "El jardín de los senderos que se bifurcan" y "El milagro secreto".

La investigación sobre la comprensión de textos es frondosa y aun así los resultados siguen siendo tentativos y preliminares con respectos a una efectiva solución a la poca o nula comprensión de lectura. Lamentablemente hay un marcado sesgo a no tomar en cuenta los textos narrativos, subestimando su complejidad y obviando o estereotipando su relevancia. Quienes los abordan, reducen al texto narrativo a mero entretenimiento sin análisis ni verificación de la comprensión. Y quienes los estudian, prefieren tomar textos argumentativos o expositivos asumiendo un rigor superior. Desaprovechando el enclave de lo narrativo en las comunicaciones actuales, ya que son la base de estos tiempos en la comunicación. Y también no aprovechando textos narrativos que siendo cortos de extensión son complejos como las obras de Borges.

Por último, al escoger un diseño cuasi experimental, la presente investigación propone un corte transversal de la investigación, asumiendo que solo en las tesis (y muy poco en artículos) se observan el abordaje de experimentos que busquen influenciar significativamente la comprensión de textos. Asimismo, hay un poco abordaje de investigaciones específicas con estudiantes universitarios en relación con textos narrativos. Por todo ello, este trabajo pretende contribuir con resultados que motiven a seguir bregando por una sociedad que comprenda lo que lea, ya que así también comprenderá lo que está viviendo.

\section{Lectura y comprensión en entornos digitales}

Leer en físico o en un entorno digital crea una gran disyuntiva pedagógica en su uso y aprovechamiento en ambos soportes. Los textos físicos son concretos y fijos al entorno que lo facilita; mientras que el digital es intangible y virtual (Cordón, 2016). Estas diferencias inquietaron a los intelectuales que pensaron podría precarizarse no solo la lectura en sí, sino su comprensión. Sin embargo, Piovano y Burin (2014) no encontraron diferencias en la comprensión. A pesar de ello, usuarios y especialistas han marcado la gran diferencia cualitativa entre leer en físico y en un entorno digital cuando se trata de textos voluminosos. En efecto, en este tipo de lecturas la luz monótona de las pantallas ocasiona un cansancio visual en el lector. Incluso puede provocar "ojo seco" por su excesiva exposición. Asimismo, si la lectura se da en dispositivos móviles, esta reduce la visión holística de toda una página como en una lectura en libro concreto. Aun así, los estudios fueron decantando estas desventajas a simples peculiaridades, entendiendo que cada una de ellas tenían características propias y por tanto sus ventajas y desventajas intrínsecas.

Para algunos investigadores pro digitales, la lectura virtual en dispositivos electrónicos implicaba mayor interacción y dinamismo, que leer en simples páginas. Pero 
que no por ello carecían de elementos disruptivos. Y que, para evitar la sobrecarga cognitiva, el lector digital debía contar con competencias específicas para aprovechar todas las ventajas del texto virtual y aminorar sus desventajas (Cordón, 2016). En este mismo sentido, Romero (2014) manifiesta que es necesario que los lectores sean lectoespectadores eficaces que se relacionen con el escrito de una manera más fluida, efectiva y constante. Competencias como el diseño, la búsqueda y la navegación (Fajardo et al; Wu, 2014 citado en Burin, Coccimiglio, González y Bulla, 2016). Es por ello que la presente investigación ha enfatizado el diseño de las actividades que toman en cuenta lo interactivo y los conocimientos previos (Martínez, Marrujo, Perillo, González y Burin, 2019) así como la búsqueda del conflicto cognitivo para redondear la comprensión.

\section{Comprensión de textos narrativos}

La comprensión de un texto se entiende como un ejercicio mental que implica esfuerzo y el planteamiento de una finalidad intelectiva (Ramos, 2007) y una suerte de interacción entre lector $\mathrm{y}$ texto en ir construyendo activamente una determinada intelección (Blandón y Restrepo, 2015). Si el texto a comprender es del tipo narrativo, se tomará en cuenta la sucesión de sucesos, la situación y ambientación de cada personaje involucrado en una historia (Calderón y Quesada, 2016) a lo largo de una secuencia temporal (Saldarriaga, 2017). La narración es el tipo de texto holístico por antonomasia, ya que dentro de él están otros tipos de texto tanto en la actualidad como desde los inicios de los tiempos (Barthes, 1996).

Es importante subrayar que dentro de los textos más leídos tenemos a las novelas y a los cuentos. Siendo las primeras, las más voluminosas y las que se relacionan por tanto a un soporte físico por antonomasia. A diferencia de los segundos, que se pueden acceder con mayor facilidad tanto de manera física, pero sobre todo digital, por su corta extensión. Sin embargo, no se están usando como se debiera, ya que, según Salazar, et al (2015) solo se establece el $6 \%$ para revisión de narraciones cortas como cuentos.

Los textos narrativos cortos son por lo general empleados como inducción a lecturas posteriores más complejas y de más alcance. Se ha empleado en la transición del lector bisoño al lector más habituado (Acevedo, Cabana y Martínez, 2013; Arciniega y Chiriboga, 2015). Pero no se debería reducir a las narraciones como simples nexos de lo simple a lo complejo o de lo breve a lo extenso. Ya que pueden ser cortos y complejos en sí mismos. Además de ser en estos tiempos los vehículos comunicativos más usados, sobre todo en las plataformas digitales.

Es por ello, que esta investigación ha elegido los cuentos "El milagro secreto" y "El jardín de senderos que se bifurcan”. Dos narraciones cortas de un autor como Borges, que es considerado un escritor que se caracteriza por su concisión y complejidad.

Nivel de impacto de los programas y aplicativos digitales

Para trabajar tanto la lectura y su posterior comprensión, se optó por una estrategia didáctica que tomara en cuenta los niveles de impacto de la tecnología en lo educativo. Según Puentedura (2006); López (2015) como se citó en Mendoza, García y Guzmán (2018), este impacto se puede dar: (1) mejorando y transformando con una sustitución 
moderada de lo físico por lo digital; (2) aumentando lo real en lo virtual con una sustitución directa y funcional; (3) modificando lo real en lo virtual con una sustitución directa, funcional y el empleo de estrategias didácticas específicas; y (4) modificación de lo real en lo virtual con una sustitución y el empleo de herramientas TIC en la creación de nuevos aprendizajes.

En esta investigación se optó por el tercer nivel de impacto. Ya que tanto los programas y aplicativos empleados buscaron una sustitución directa de la lectura física por una virtual de manera funcional, así como el empleo de estrategias didácticas puntuales para realizar procesos pedagógicos específicos como recojo de saberes previos, conflicto cognitivo, recuperación y organización de información, y evaluación de la comprensión lectora.

El criterio para la elección de los aplicativos y programas fueron los que resultaban más intuitivos y no implicaban sobrecarga cognitiva, ni mucha dificultad para su acceso ni sofisticación en su procesamiento; ya que, en algunas ocasiones, las herramientas TIC en vez de ser un medio, se transforman involuntariamente en un fin en sí mismas (Levis, 2016 citado en Pérez-Rodríguez y Ponce, 2012). Además de tener en la mayoría de casos, condicionamientos económicos para sus suscripciones.

Por ello, la presente investigación empleó los siguientes programas y aplicativos: Biblioteca digital Seva, Kahott, miMind, Educaplay, Goconqr, y Thatquiz. La Biblioteca digital Seva se empleó para acceder a los dos cuentos empleados en este trabajo. El Kahott se utilizó para el recojo de saberes previos y el conflicto cognitivo. El miMind fue idóneo para recuperar y organizar información en Mapas Mentales Armónicos. La plataforma Educaplay fue muy importante para el diseño y aplicación de actividades lúdicas posterior a la lectura como Sopa de letras, crucigramas y mapas interactivos. Finalmente, tanto el Goconqr como el Thatquiz sirvieron para la evaluación de las lecturas.

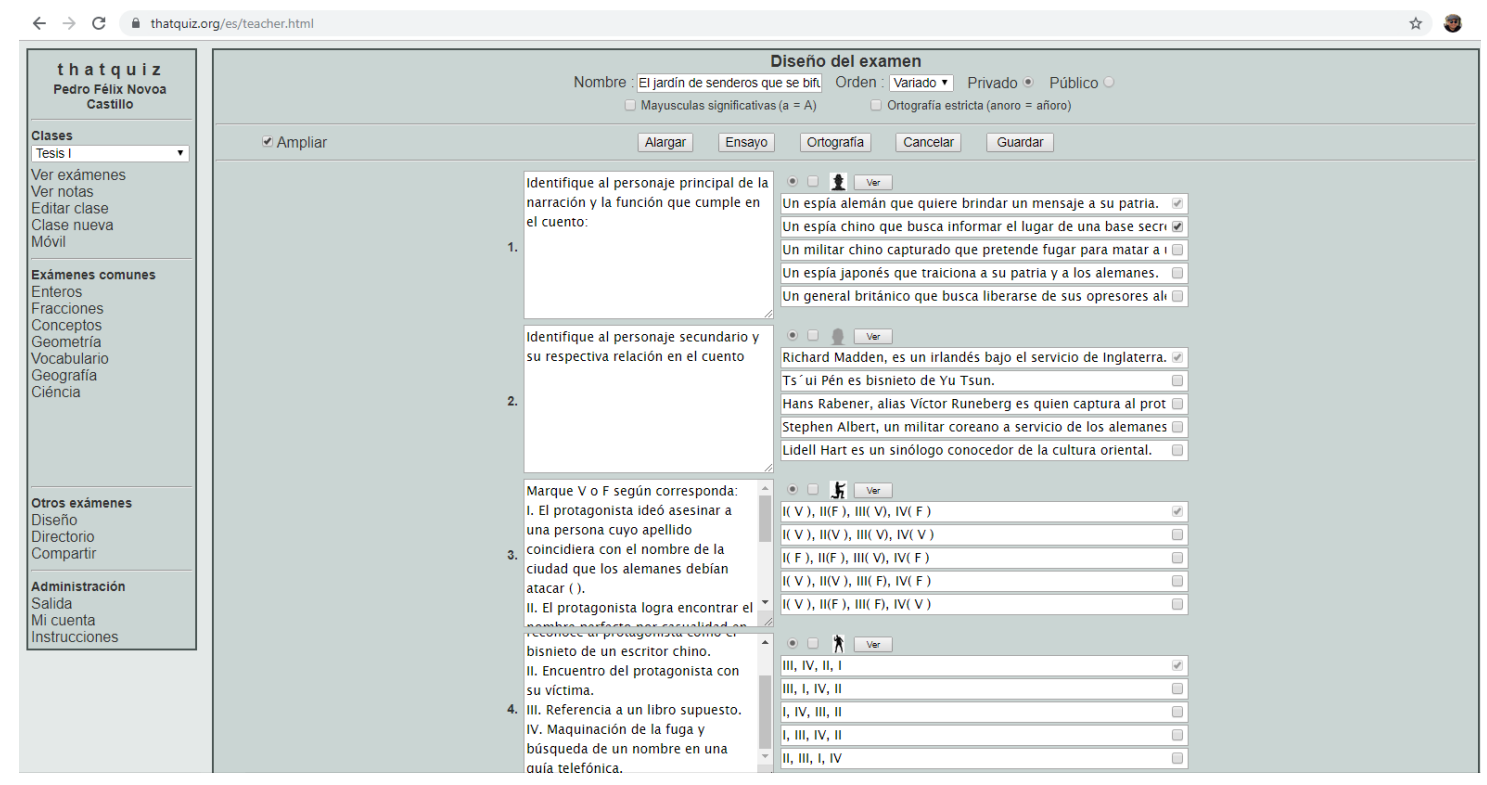

Gráfico 1. Diseño de pruebas en Thatquiz (recortado)

Es necesario anotar que el empleo de lo tecnológico en educación es todavía bajo. Salazar, et al (2015) revelaron que solo el $6 \%$ empleaba dispositivos electrónicos con fines educativos en Colombia. Hay que añadir que, debido a la pobreza, la brecha tecnológica en Latinoamérica es mayor (Rodríguez, 2005; Bravo, 2012) y precisamente por ello, la exigencia a los docentes en aprovechar al máximo los recursos disponibles. Es 
en este contexto, que las estrategias en entornos digitales se convierten en medios idóneos para influir de manera positiva en los aprendizajes.

Luego de asegurar el aprendizaje, lo inmediato es la evaluación. Para ello, este trabajo optó por las pruebas en línea. Modalidad que, según Thorne, et al (2013); y Ponce, et al (2007) constituye una alternativa que, de ser bien empleada, puede mejorar los resultados. En esta investigación se aplicó un pre test y dos pos test (uno previo y uno definitivo). Entendiendo que el hecho de realizar una evaluación adicional a manera de ensayo significa la seguridad de mejorar la comprensión total del texto leído (Benítez, Barajas, Uresti y Nallehly, 2014).

Las evaluaciones en línea, a su vez, tienen la ventaja de ser inmediatas, rápidas y con la opción de autorregulación de acuerdo a cada ritmo de aprendizaje. Los investigadores recomiendan que las evaluaciones sobre lectura apelen a un diseño contextualizado (Mon y Cervera, 2013) y que cada pregunta sobre la lectura busque una comprensión profunda y significativa (Martínez, Marrujo, Perillo, González y Burin, 2019).

\section{Mapas Mentales Armónicos en digital}

Un recurso imprescindible para la estrategia empleada en esta investigación fue el empleo de los Mapas Mentales Armónicos. Entendidos como la versión mejorada de los clásicos de Buzan (1996); ya que se elimina una característica negativa como lo abigarrado del original, y se la reemplaza por lo armónico, logrando que las ramas pierdan su desorden y ganen una distribución equilibrada y más armoniosa (Novoa, 2019; Novoa et al, 2018) manteniendo sus características más relevantes como el uso de figuras o íconos con alta carga semántica (Fernández, Vázquez y López, 2016).

Con estos organizadores de información se pudo recuperar, ordenar y jerarquizar la información recién adquirida en la lectura. Para su diseño elegimos los digitales, ya que estos presentan bondades como la organización reticular o bifurcada en tiempo real, creativa y libre (Apolo, Bayés y Hermann, 2016).

Este organizador está dentro del enfoque cognitivo que tiene últimamente mucha adhesión en la comunidad educativa (Salazar et al, 2015). Ya que, dentro de los estudios relacionados con el aprendizaje, los Mapas mentales permiten construir nuevos conocimientos a partir de la gestión, organización y jerarquización de los mismos implicando relaciones y sentidos que los vinculan, creando una suerte de imagen mental de un contenido específico (Campos, 2005; Villalustre y Del Moral, 2010). Asimismo, se considera didácticamente a los Mapas Mentales como flexibles y efectivos en la tarea de consolidar aprendizajes (Ortega y Zósimo, 2012), ya que estos organizadores visuales permiten organizar segmentos específicos de conocimiento de manera interrelacionada.

Para realizar estos esquemas se empleó en esta investigación la plataforma miMind. 


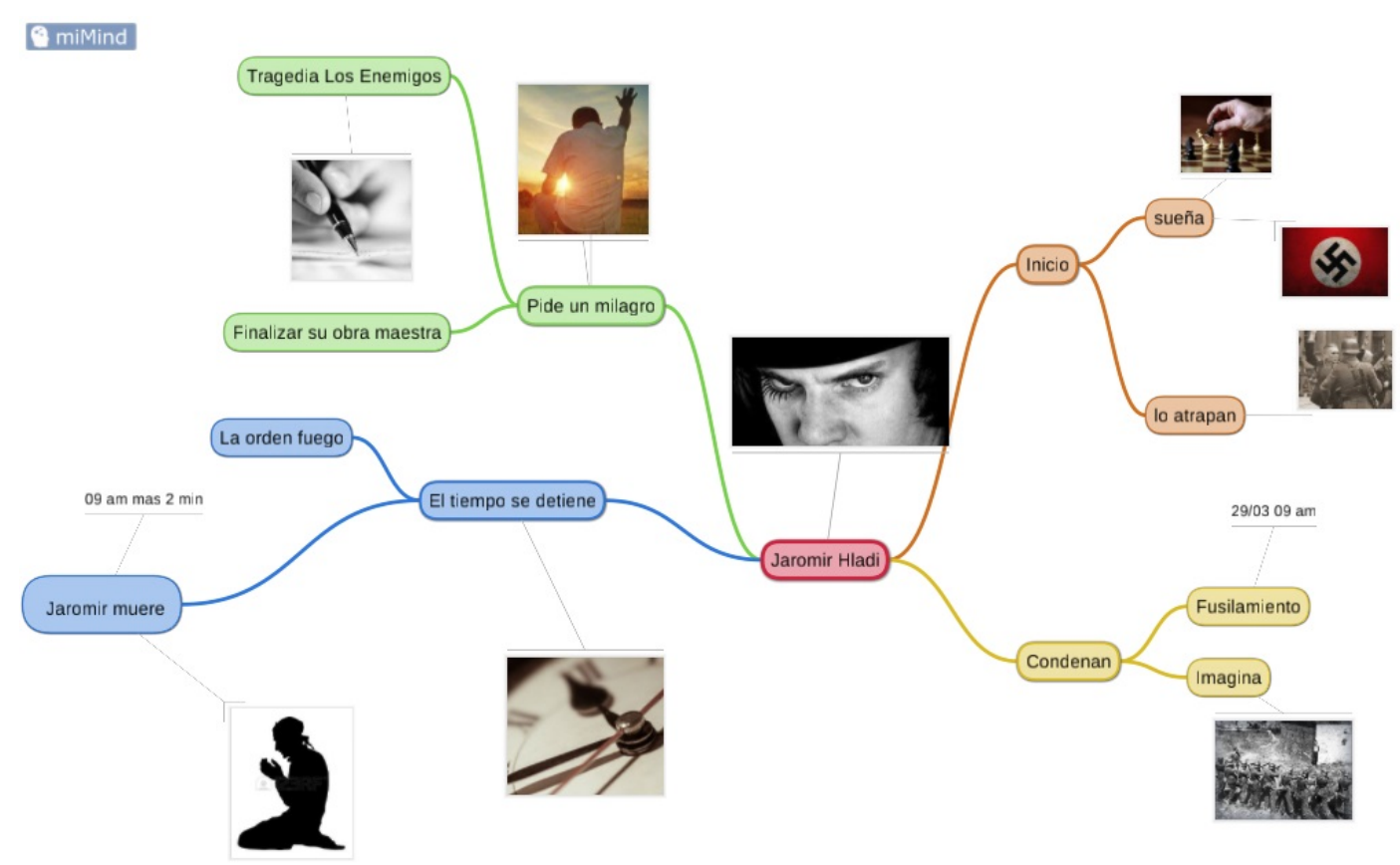

Gráfico 2. Organización de lo leído en un Mapa Mental Armónico en digital (miMind)

\section{Objetivos e hipótesis}

La investigación se propuso demostrar que ciertos programas y aplicativos de naturaleza práctica y sencilla, empleados como estrategia digital por parte de los docentes, influyen de manera positiva en la comprensión lectora de textos narrativos (objetivo general). Así como en sus dimensiones textual, inferencial y criterial (objetivo específico).

Debido al método hipotético deductivo del presente trabajo se plantearon las siguientes hipótesis:

1. La estrategia digital mejora la comprensión de textos narrativos (Hipótesis general).

2. La estrategia digital mejora la comprensión textual, inferencial y criterial de textos narrativos (Hipótesis específica).

\section{Método}

Se trabajó con un diseño de investigación experimental del tipo cuasi experimental de corte transversal, debido a la necesidad de comprobar los efectos de una estrategia basada en el empleo de ciertos programas y aplicativos digitales y considerando que la experiencia debía ser de corte transversal. Asimismo, con este diseño metodológico se puede además de experimentar la estrategia medir su impacto.

\section{Participantes}

La investigación se enfocó en una población de 1580 estudiantes de la Facultad de Educación de la UNMSM, de los cuales se tomó una muestra de 310 participantes (Grupo control: 160 y Grupo experimental: 150) de edades oscilantes entre los 18 a 27 años, a través de un muestreo probabilístico simple (Malhotra, 2008). Los seleccionados firmaron 
un documento de consentimiento informado y se les dio su respectiva devolución de resultados.

\section{Instrumentos/Materiales}

Se emplearon dos narraciones de Borges disponibles en línea (Ciudad Seva): "El jardín de los senderos que se bifurcan" como lectura para pre test y "El milagro secreto" como lectura del pos test. Ambos textos de similar complejidad, aunque diferente extensión de palabras (3747 y 2242). Por ello se asignó un tiempo de lectura de 37 minutos para el primer cuento y 22 minutos para el segundo.

Se aplicaron tres pruebas tipo PISA para la comprensión de textos narrativos (Basadas en Novoa, et al, 2018; Novoa, 2019) donde a través de preguntas de alternativa múltiple se recogía información de comprensión literal, inferencial y criterial: un pre test en la plataforma Thatquiz, un primer pos test en el entorno Goconqr y un último pos test en Thatquiz.

Se empleó un breve cuestionario de dos minutos en Kahoot, antes de la lectura; y luego de la lectura un Mapa Mental Digital a través de la plataforma miMind, durante 15 minutos; se realizó tres actividades lúdicas a través de la plataforma Educaplay: un crucigrama (10 minutos), una sopa de letras (10 minutos) y un mapa interactivo (5 minutos).

\section{Procedimiento}

En la primera clase se efectúa la firma del consentimiento informado, se procede a la lectura de la lectura "El jardín de los senderos que se bifurcan" y concluida esta, se aplica el pre test tanto al grupo de Control como al grupo Experimental.

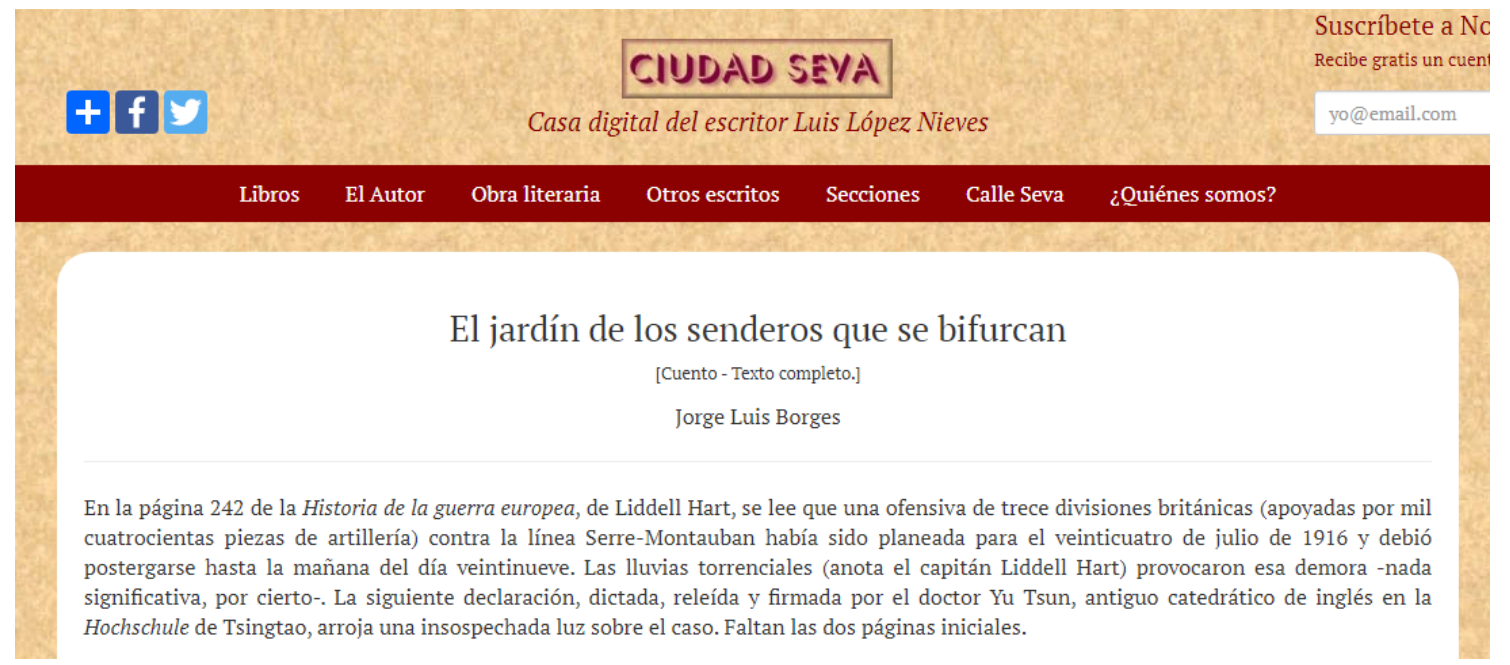

Figura 1. Cuento del Pre test (recortado) 


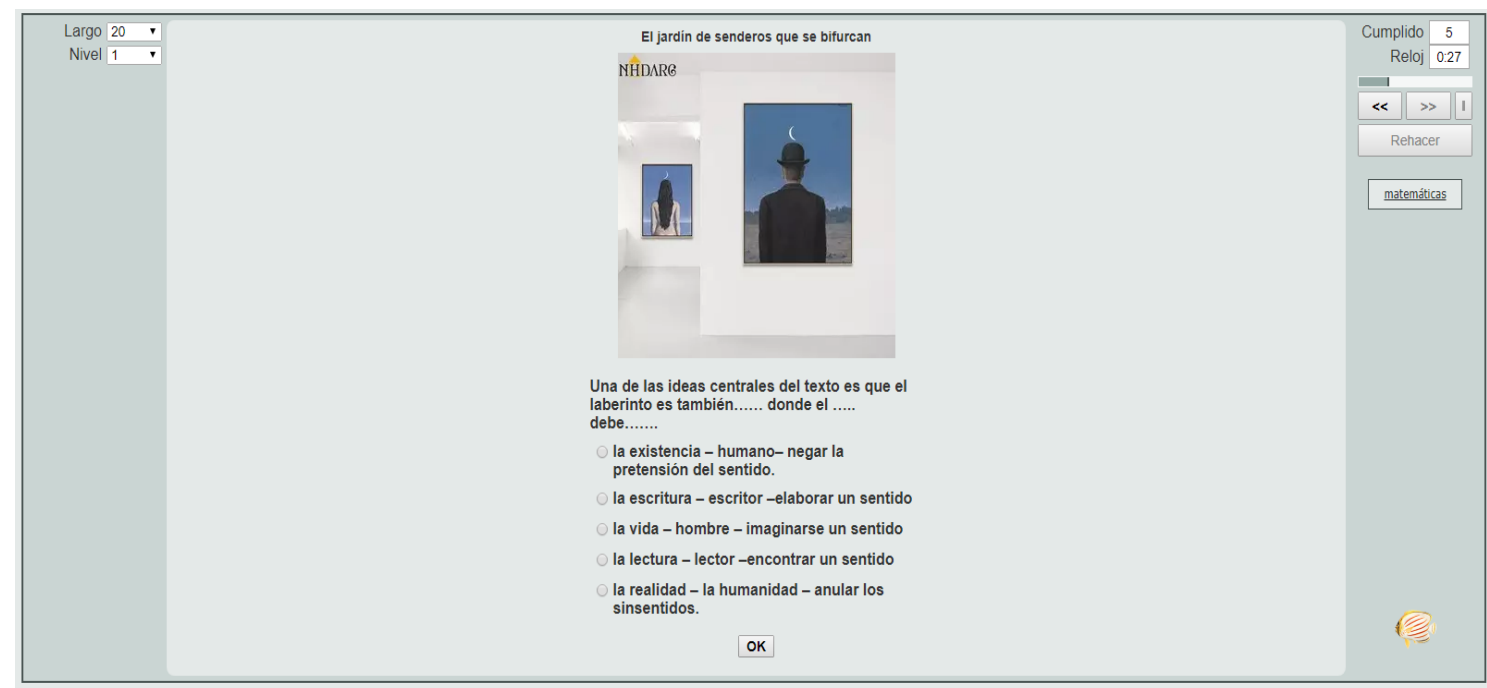

Figura 2. Pre test (recortado)

En la segunda clase, se desarrolla con el grupo Experimental una recuperación de los saberes previos y se busca el conflicto cognitivo a través de un breve cuestionario Kahoot, donde las dos primeras preguntas son para el recojo de saberes previos, importante para anclar y relacionar con información nueva; y las dos últimas para provocar un conflicto cognitivo a partir de la reflexión sobre el tiempo. Tema crucial del cuento que se irá a leer. El conflicto se plantea a partir de la afirmación de que el hombre está o no sumido ante el tiempo. Se da como verdadera la aseveración, pero se aprovecha para ocasionar un desequilibrio en la adquisición de nuevos aprendizajes (Campione, Brown y Ferrara, 1987; Piaget, 1985; Siegler y Richards, 1989 como se citó en Ugartetxea, 1996).

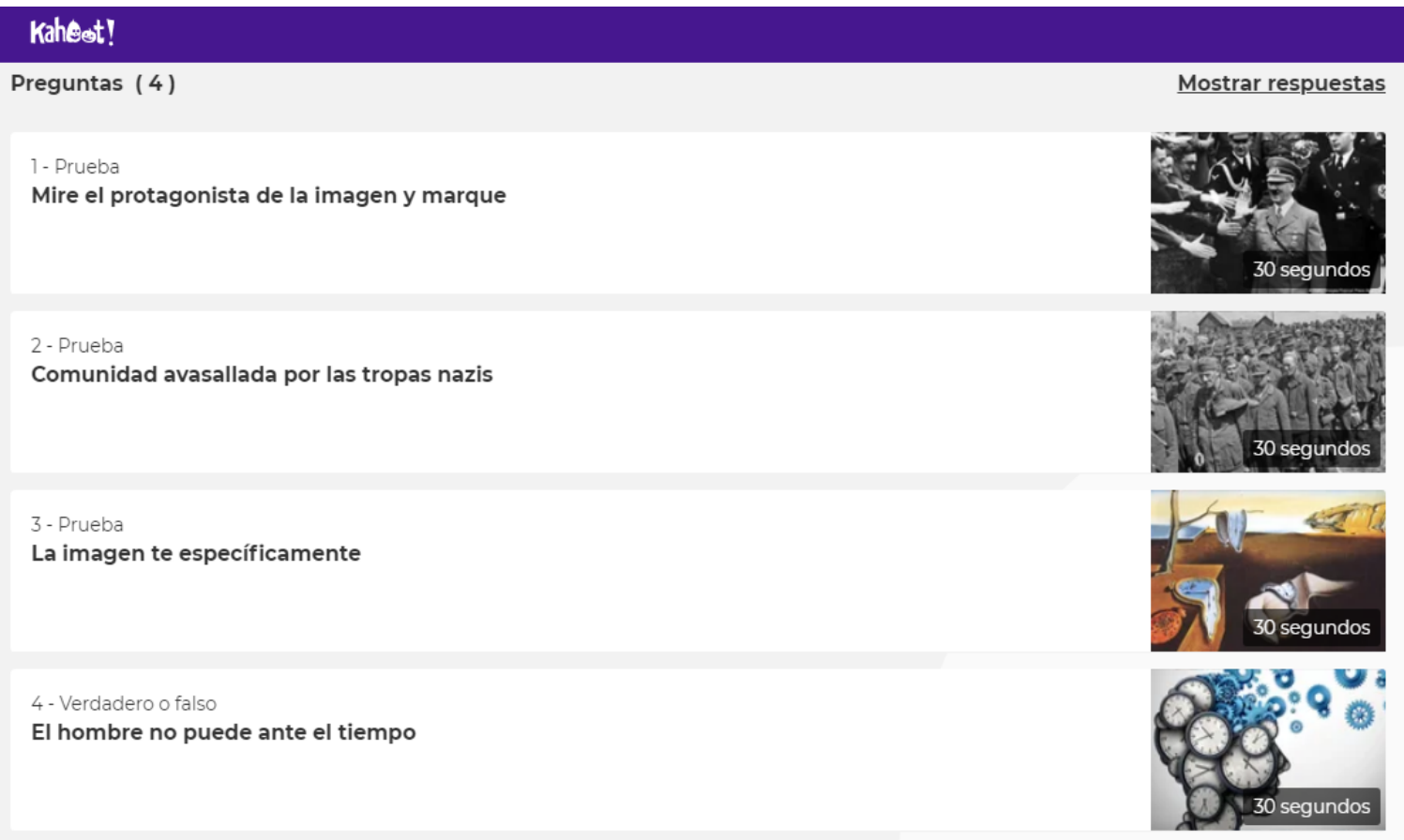

Figura 3. Breve cuestionario Kahoot (recortado) 
Terminada la dinámica del Kahoot, se procede a la lectura de "El milagro secreto". Terminada esta, se realiza el primer pos test. Se socializa algunas reflexiones de las preguntas que hayan provocado dudas en los estudiantes. Se realiza una organización de información a través de la elaboración de un Mapa Mental Armónico en la plataforma miMind. Se continúa con tres actividades lúdicas que servirán como reforzadoras de conocimientos nuevos: un crucigrama, para aludir temáticas relacionadas; una sopa de letras, para reforzar palabras clave y un mapa interactivo que asegurará la secuencia narrativa del cuento leído. Incorporar lo lúdico en las estrategias para incentivar la comprensión lectora es importante, ya que permite aminorar la complejidad y dificultad del proceso de comprensión (Farrach, 2016).

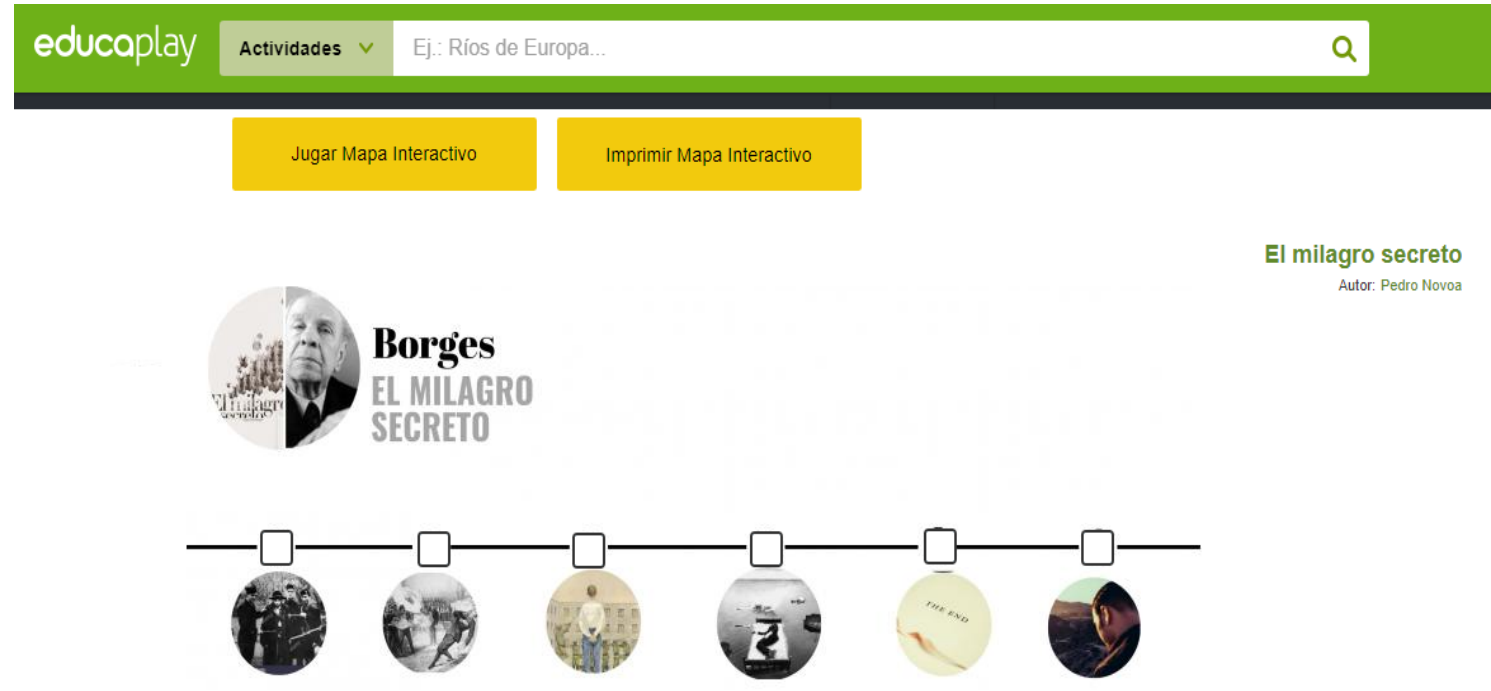

Figura 4. Mapa interactivo Educaplay (recortado)

Finalmente se toma el último pos test.

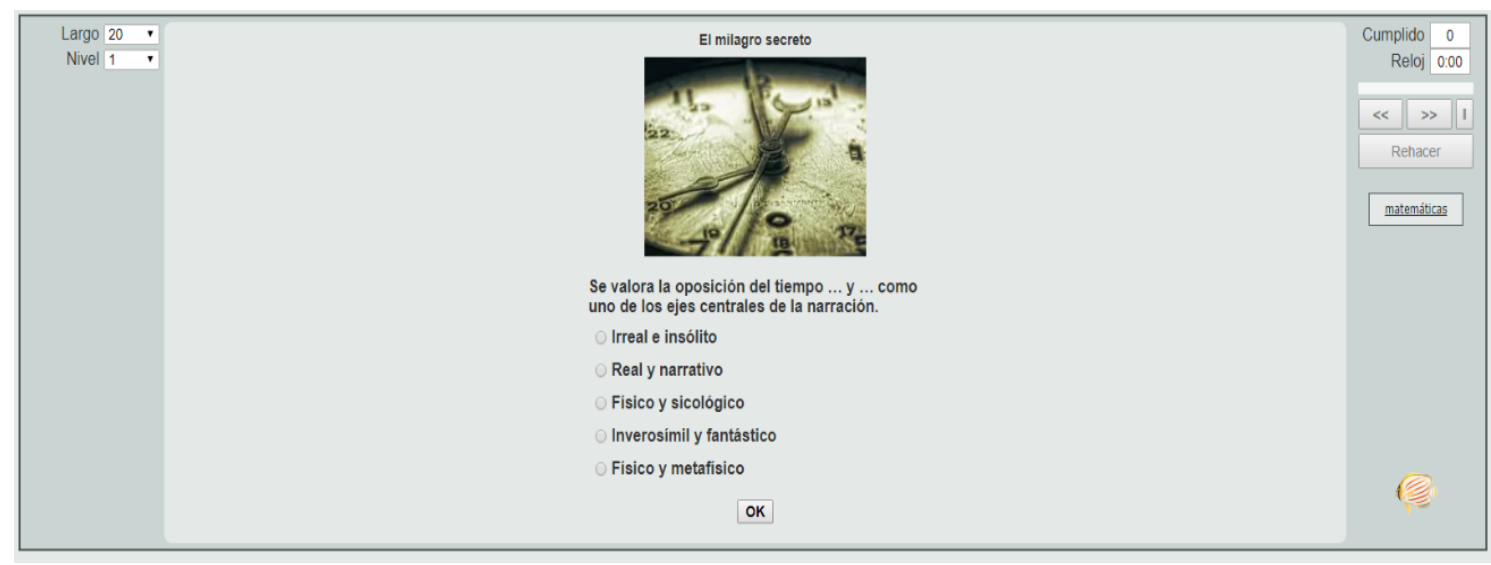

Figura 5. Último pos test Thatquiz (recortado) 


\section{Resultados}

Se analizó las notas de comprensión de textos narrativos y se les aplicó la prueba de normalidad.

Tabla 1. Prueba de normalidad de Comprensión de textos narrativos

\begin{tabular}{ccccc}
\hline Kolmogorov-Smirnov $^{\mathbf{a}}$ & test & Estadístico & gl & Sig. \\
\hline Comprensión de textos & pre control & .153 & 160 & .000 \\
narrativos & pre experimental & .175 & 150 & .000 \\
& pos control & .140 & 160 & .000 \\
& pos experimental & .174 & 150 & .000 \\
\hline
\end{tabular}

a. Corrección de significación de Lilliefors

Y al ser el p valor de significancia menor que .005, se determinó que los datos presentaban una distribución no normal. Debido a esto, se estableció como prueba no paramétrica para la posterior contrastación de hipótesis la U the Mann Whitney.

\section{Resultados descriptivos}

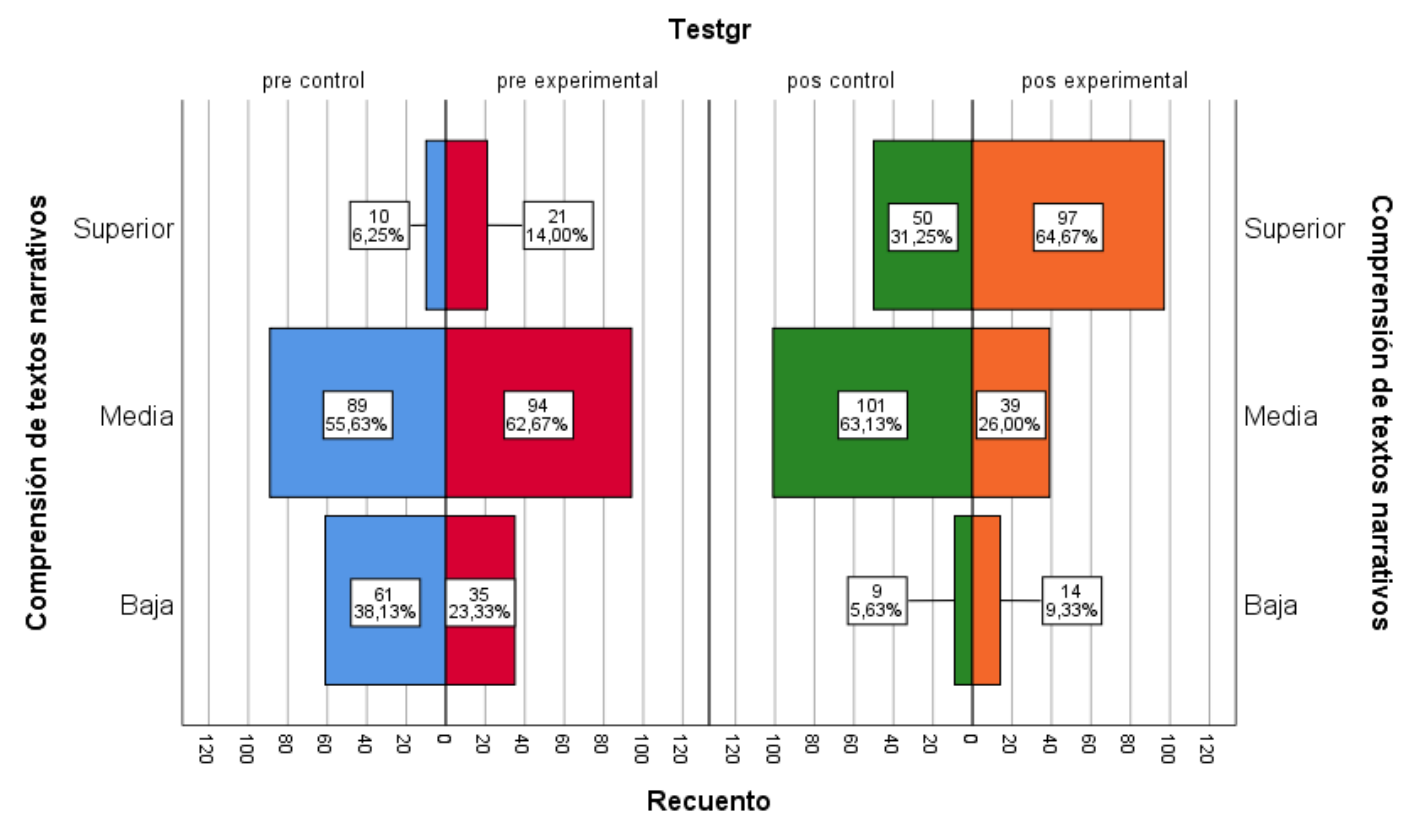

Gráfico 3. Resultado de las Pruebas de Comprensión de textos narrativos

Se observa en el Gráfico 3 que en el pre test de Comprensión de textos narrativos tanto el grupo control como el grupo experimental estuvieron en condiciones similares, aunque con pequeñas diferencias. En cambio, en el pos test, estas diferencias se remarcaron en favor del grupo experimental; ya que un importante porcentaje del nivel bajo ascendió al nivel medio; así como otro gran porcentaje se elevó del medio al nivel superior. 


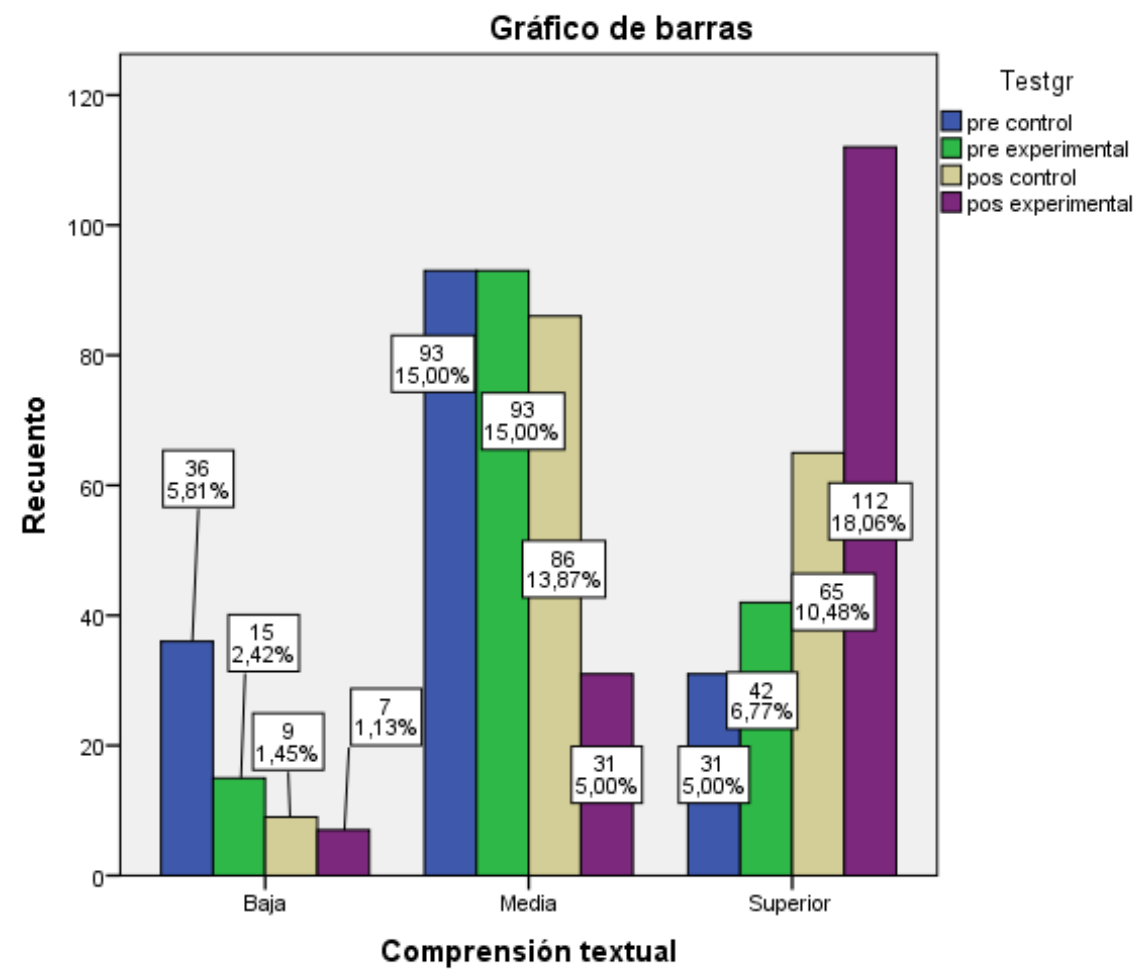

Gráfico 4. Comprensión textual de textos narrativos por pruebas

En el Gráfico 4 se observa que la dimensión Comprensión textual muestra en el Pre test ligeras diferencias en los niveles bajo y superior. Mientras que en el pos test, hay una marcada diferencia en favor del grupo Experimental.

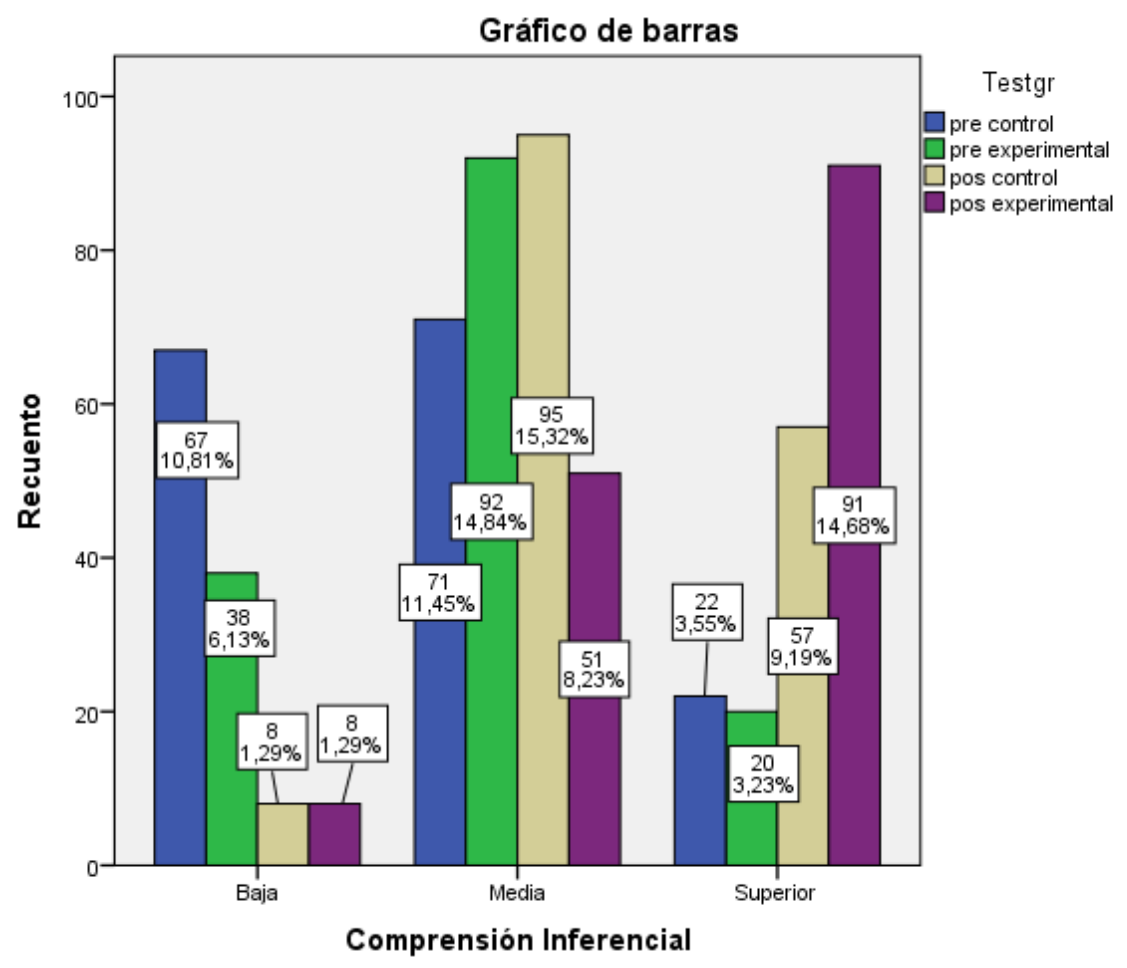

Gráfico 5. Comprensión inferencial de textos narrativos por pruebas 
En el Gráfico 5 se observa que la dimensión Comprensión inferencial muestra en el Pre test ligeras diferencias en los niveles bajo, medio y superior. Mientras que en el pos test, hay una marcada diferencia en favor del grupo Experimental en el nivel superior, pero ninguna en el nivel bajo y más bien una gran ventaja del Grupo de control en el nivel medio.

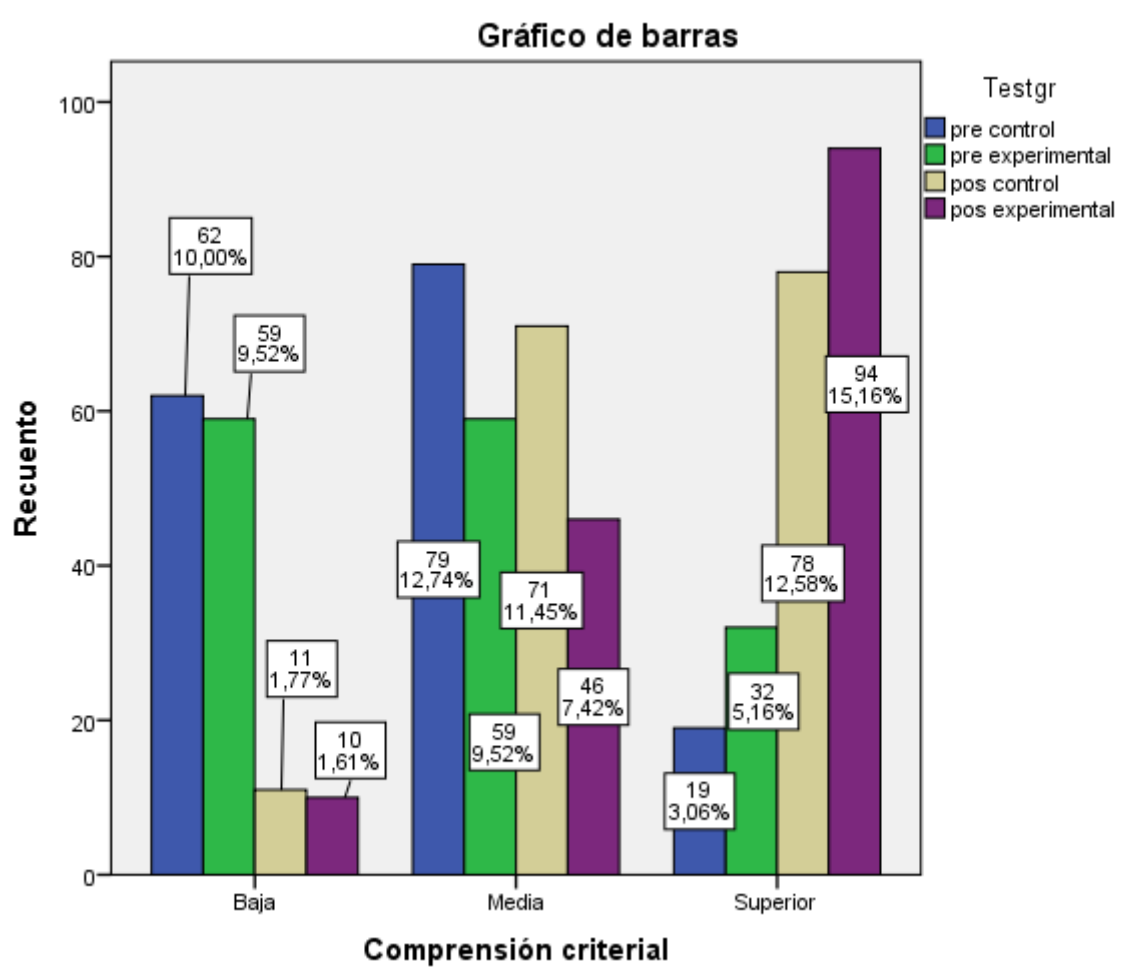

Gráfico 6. Comprensión criterial de textos narrativos por pruebas

En el Gráfico 6 se observa que la dimensión Comprensión criterial muestra en el Pre test ligeras diferencias en los niveles bajo, medio y superior. Mientras que en el pos test, hay una marcada diferencia en favor del grupo Experimental en el nivel superior; más no en el medio donde el Grupo control tiene predominancia.

\section{Resultados inferenciales}

Prueba de hipótesis general:

$\mathrm{H}_{0}$ : Las estrategias de aplicación digital no mejoran la comprensión de textos narrativos

$\mathrm{H}_{\mathrm{i}}$ : Las estrategias de aplicación digital mejoran la comprensión de textos narrativos 
Tabla 2. Prueba U de Mann-Whitney para probar la hipótesis general

\begin{tabular}{|c|c|c|c|c|c|c|}
\hline \multicolumn{5}{|c|}{ Rangos } & \multicolumn{2}{|c|}{ Estadísticos de contraste } \\
\hline \multirow{8}{*}{$\begin{array}{l}\text { Comprensi } \\
\text { ón de textos } \\
\text { narrativos }\end{array}$} & Test y Grupo & $N$ & $\begin{array}{l}\text { Rango } \\
\text { prom. }\end{array}$ & $\begin{array}{l}\text { Suma } \\
\text { rangos }\end{array}$ & & \\
\hline & Pre Control & 160 & 162.69 & 26030.00 & $\begin{array}{c}\text { Ude Mann-Whitney } \\
\text { W de Wilcoxon }\end{array}$ & $\begin{array}{l}10850.000 \\
22175.000\end{array}$ \\
\hline & Pre Experimental & 150 & 147.83 & 22175.00 & Z & $-1,479$ \\
\hline & & & & & $\begin{array}{l}\text { Sig; p. Asintót. } \\
\text { (bilateral) }\end{array}$ & .139 \\
\hline & Pos Control & 160 & 134.88 & 21580.00 & Ude Mann-Whitney & 8700.000 \\
\hline & & & & & $W$ de Wilcoxon & 21580.000 \\
\hline & Pos Experimental & 150 & 177.50 & 26625.00 & Z & -4.216 \\
\hline & & & & & $\begin{array}{c}\text { Sig; } p \text {. Asintót. } \\
\text { (bilateral) }\end{array}$ & .000 \\
\hline
\end{tabular}

Se observa en la tabla 2 que en el pre test de Comprensión de textos narrativos los rangos del Grupo de Control y el Grupo Experimental no son tan diferenciados. Y que tanto el valor $p$. de significancia (mayor que $\alpha=.05$ ) y el valor $Z$ (mayor que el límite crítico -1.96) corroboran que no existen diferencias significativas entre ambos grupos. Sin embargo, en el Postest, sí hay diferencia marcada en los rangos. Además, se aprecia que el p. de significancia (mayor que $\alpha=.05$ ) y el valor $Z$ (menor que el límite crítico -1.96) corroboran que existen diferencias significativas entre ambos grupos. Por tanto, se rechaza la hipótesis nula y se concluye que Las estrategias de aplicación digital mejoran significativamente la Comprensión de textos narrativos.

Prueba de hipótesis específica

$\mathrm{H}_{0}$ : Las estrategias de aplicación digital no mejoran la comprensión textual, la comprensión inferencial y la comprensión criterial de textos narrativos.

$\mathrm{H}_{\mathrm{i}}$ : Las estrategias de aplicación digital no mejoran la comprensión textual, la comprensión inferencial y la comprensión criterial de textos narrativos.

Tabla 3. Prueba U de Mann-Whitney para probar la hipótesis especifica

\begin{tabular}{|c|c|c|c|c|c|c|}
\hline \multicolumn{5}{|c|}{ Rangos } & \multicolumn{2}{|c|}{ Estadisticos de contraste } \\
\hline \multirow{8}{*}{$\begin{array}{l}\text { Comprensi } \\
\text { ón textual } \\
\text { de textos } \\
\text { narrativos }\end{array}$} & Test y Grupo & $N$ & $\begin{array}{l}\text { Rango } \\
\text { prom. }\end{array}$ & $\begin{array}{l}\text { Suma } \\
\text { rangos }\end{array}$ & & \\
\hline & Pre Control & 160 & 162.50 & 25999.50 & $\begin{array}{c}U \text { de Mann-Whitney } \\
W \text { de Wilcoxon }\end{array}$ & $\begin{array}{l}10880.500 \\
22205.500\end{array}$ \\
\hline & Pre Experimental & 150 & 148.04 & 22205.50 & Z & -1.455 \\
\hline & & & & & $\begin{array}{l}\text { Sig; p. Asintót. } \\
\text { (bilateral) }\end{array}$ & .146 \\
\hline & Pos Control & 160 & 144.32 & 23091.00 & Ude Mann-Whitney & 10211.000 \\
\hline & & & & & $W$ de Wilcoxon & 23091.000 \\
\hline & Pos Experimental & 150 & 167.43 & 25114.00 & $Z$ & -2.306 \\
\hline & & & & & $\begin{array}{c}\text { Sig; } p \text {. Asintót. } \\
\text { (bilateral) }\end{array}$ & .021 \\
\hline & Test y Grupo & $N$ & $\begin{array}{l}\text { Rango } \\
\text { prom. }\end{array}$ & $\begin{array}{l}\text { Suma } \\
\text { rangos }\end{array}$ & & \\
\hline & Pre Control & 160 & $\begin{array}{r}154.57 \\
49\end{array}$ & 24730.50 & U de Mann-Whitney & 11850.500 \\
\hline
\end{tabular}




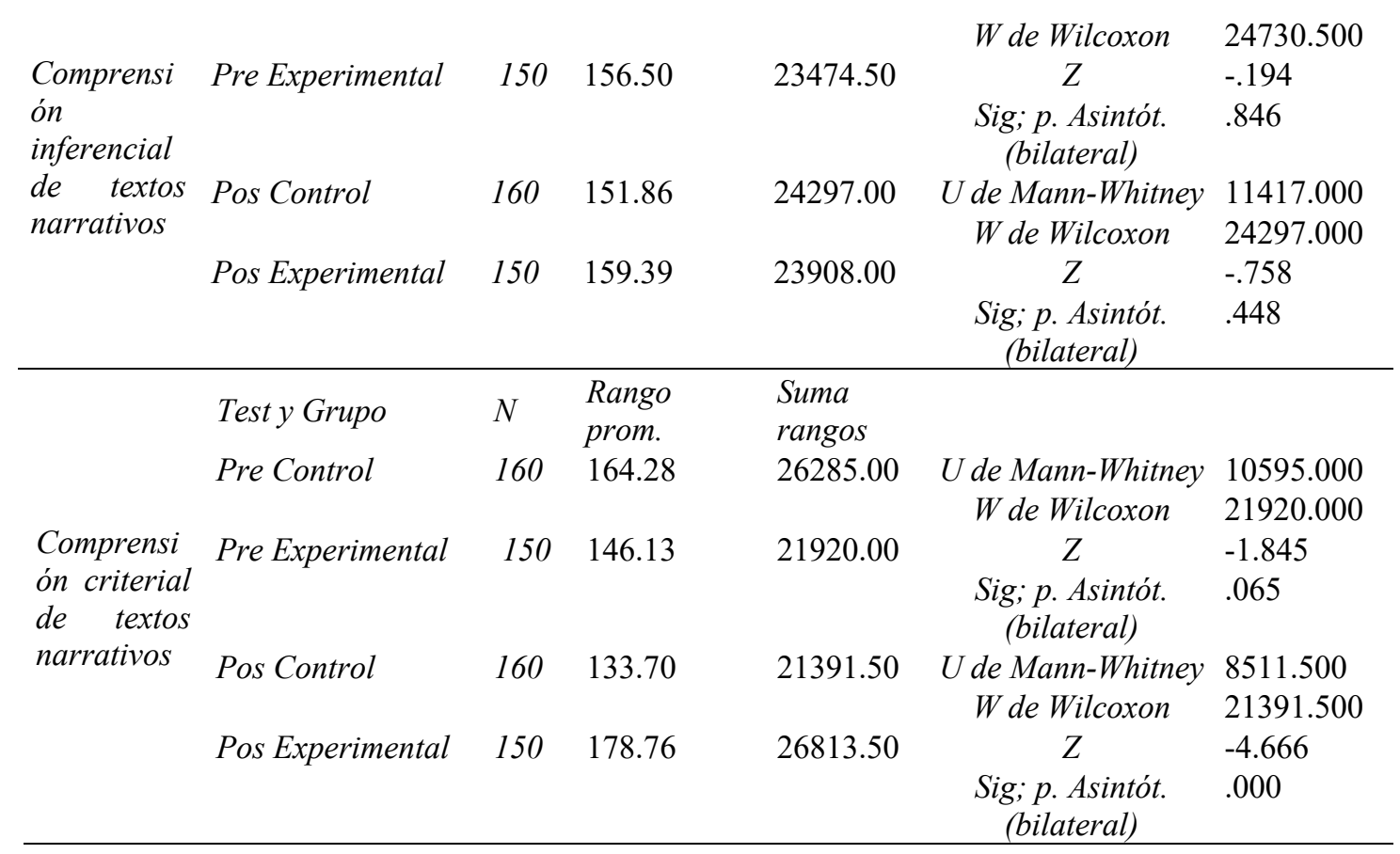

Se observa en la tabla 3 que en el pre test de Comprensión textual, Comprensión inferencial y Comprensión criterial de textos narrativos los rangos del Grupo de Control y el Grupo Experimental no son tan diferenciados. Y que tanto el valor p. de significancia (mayor que $\alpha=.05$ ) y el valor $Z$ (mayor que el límite crítico -1.96) corroboran que no existen diferencias significativas entre ambos grupos. Sin embargo, en el Postest, sí hay diferencia marcada en los rangos de Comprensión textual y Comprensión criterial de textos narrativos. Además, se aprecia que el p. de significancia (menor que $\alpha=.05$ ) y el valor $\mathrm{Z}$ (menor que el límite crítico -1.96) corroboran que existen diferencias significativas entre ambos grupos. A excepción de la Comprensión inferencial que mantuvo tanto sus rangos similares y que tanto su valor $\mathrm{p}$. de significancia y valor $\mathrm{Z}$ se mantuvieron también altos, por ello la influencia en esta dimensión no es significativa. Por todo lo explicado, se rechaza de manera parcial la hipótesis nula y se concluye que Las estrategias de aplicación digital mejoran significativamente la Comprensión textual y Comprensión criterial de textos narrativos.

\section{Discusión}

En la presente investigación se puso a prueba algunas actividades en entornos digitales con el objetivo de que los alumnos lean y comprendan textos narrativos de corta extensión. La aplicación de este tipo de estrategia ha resultado de gran impacto en la comprensión de textos narrativos. Estos resultados entran en dialógica controversia con lo hallado por Pulgar (2016), quien también empleó un diseño cuasi experimental, para corroborar la significatividad de la incorporación de herramientas TIC como estrategia para mejorar la comprensión de textos narrativos en estudiantes universitarios. Y concluyó que el efecto era de un $76.9 \%$ en la comprensión lectora general al término de su investigación cuasi experimental. La incidencia en comprensión literal fue de 12.8 al 56\%; 7.7 al $66 \%$ en la comprensión inferencial; y 23.1 al $41 \%$ en la comprensión criterial. Resultados significativamente más altos de los hallados en el presente trabajo, pero que estarían signados por cierto sesgo en el procesamiento de datos al tener una distribución 
normal y por ende el empleo de pruebas no paramétricas como la T de Student. Asimismo, se observa que en el programa existe textos que no son narrativos, sino expositivos; y que a su vez el nivel de dificultad de las lecturas es disímil en su complejidad (el autor escoge textos muy cortos o fragmentos, mientras que en esta investigación se eligió cuentos completos de Borges).

Otro referente inmediato es la investigación realizada por Novoa, et al (2018), quienes encuentran una influencia significativa empleando la versión en físico de la variante digital empleada en este trabajo: el mapa mental armónico. En dicha investigación se logra una significatividad $(\mathrm{p}=.000)$, comprobando la hipótesis de trabajo, de que los Mapas Mentales Armónicos influyen significativamente en la comprensión de textos narrativos.

La importancia de añadir a las estrategias pedagógicas elementos digitales como el caso de Millalén (2015) quien realizó una investigación cuasi experimental para comprobar los efectos de la multimodalidad en la comprensión de textos narrativos en estudiantes universitarios entra en convergencia con lo hallado en esta investigación. El autor demostró que el grupo experimental sacó un puntaje de 55.6 y el grupo control de 38,8 en comprensión general. Esta ventaja fue verificada por la prueba $\mathrm{T}$ de Student $(\mathrm{t}=6.381)$ y una significancia $\mathrm{p}=.000$. Aceptando con esto su hipótesis de trabajo al igual que en esta investigación. Aunque hay que anotar que el texto era en inglés y orientado a una segunda lengua.

En este mismo contexto de aprovechamiento de entornos TIC, Hernández (2016) empleó un enfoque mixto para comprobar que la comprensión de textos narrativos mejora a través de una biblioteca digital y una plataforma virtual (Edmodo). El autor comprobó que existió una mejora relevante en los niveles sintáctico, semántico y pragmático. En este caso, la presente investigación coincide también en la influencia positiva, pero no en los instrumentos empleados, ni en las dimensiones, ya que el presente trabajo optó por las canónicas que son comprensión literal, inferencial y criterial.

Con relación a la lectura y comprensión en entornos digitales, el presente trabajo encontró mejores resultados en el grupo experimental que tuvo una permanente actividad en entornos digitales. Estos hallazgos complementan los resultados de Rodríguez y Vargas (2017), quienes encontraron ligeras ventajas en la interpretación de textos narrativos de los participantes que emplearon cuadernos digitales con los que emplearon entornos tradicionales.

En conclusión, la presente investigación brinda la posibilidad de realizar un conjunto de estrategias combinadas tanto antes y después de la lectura de un texto narrativo y poder mejorar los resultados de su comprensión. Estas estrategias por lo general son intuitivas, flexibles y sobre todo activas. Y constituyen una vertiente aún no estudiada a profundidad sobre la comprensión de textos narrativos mediados por estrategias digitales en el ámbito universitario. 


\section{Conclusiones}

Se comprobó que el empleo de una estrategia digital basada en el empleo de programas y aplicaciones virtuales de manera transversal (uno o dos días) tiene un efecto significativo en la comprensión de textos narrativos.

Se evidenció que hubo mejora en las dimensiones de comprensión textual y comprensión criterial, pero que no se consiguió la significancia en la comprensión inferencial, muy probablemente por el hecho de que exista un apresuramiento en las deducciones de los participantes, situación muy común en la juventud de estos tiempos.

Se demostró que el uso de programas y aplicativos digitales de empleo intuitivo y de poca complejidad e intromisión en las actividades educativas, tienen buenos resultados tanto en su aplicabilidad como en los resultados que evidencian mejoría.

\section{Referencias}

Acevedo, V. M., Cabana P. y Martínez V. L (2013). Estrategias pedagógicas para el desarrollo de procesos lectores y escriturales orientados a la articulación entre el nivel preescolar y la básica primaria (Tesis Doctoral). Recuperado de http://hdl.handle.net/11227/5364

Apolo, D., Bayés, M. y Hermann, A. (2016). Cambios educativos en los procesos de lectura digital: la pedagogía del ciberespacio como estrategia de procesamiento de contenidos en la era de internet. Revista de Estudios para el Desarrollo Social de la Comunicación, (12), 222. Recuperado de http://dx.doi.org/10.15213/redes.n12.p222

Arciniega T. R. y Chiriboga L. J. (2015). Comprensión lectora de fábulas para la formación de valores enfocados en el buen vivir en los alumnos del cuarto grado de las escuelas Mercedes Troya de Suárez y Víctor Manuel Peñaherrera de la ciudad de Ibarra en el año lectivo 2012; 2013. Propuesta alternativa (Tesis de Bachiller). Recuperado de http://repositorio.utn.edu.ec/handle/123456789/4008

Barthes, R. (1966). Introducción al análisis estructural del relato. Tiempo Contemporáneo: Buenos Aires, Argentina.

Benítez, L., Barajas, V., Uresti, H. y Nallehly, I. (2014). Efecto de la aplicación de una estrategia de comprensión de lectura en un entorno virtual. Revista electrónica de investigación educativa, 16(3), 71-87. Recuperado de http://www.scielo.org.mx/scielo.php?pid=S1607$40412014000300005 \mathrm{yscript}=$ sci_arttextytlng=en

Blandón, M. V. y Restrepo, L. F. (2015). La comprensión de textos narrativos: implementación de una secuencia didáctica de enfoque comunicativo, con estudiantes de grado segundo(Tesis doctoral). Recuperado de https://core.ac.uk/download/pdf/71399434.pdf

Bravo, E. (2012). Globalización, innovación tecnológica y pobreza. Aproximación a las nuevas conceptualizaciones en Latinoamérica. Espacio abierto, 21(3), 543-556. Recuperado de https://www.redalyc.org/pdf/122/12223296007.pdf

Burin, D., Coccimiglio, Y., González, F. y Bulla, J. (2016). Desarrollos recientes sobre 
habilidades digitales y comprensión lectora en entornos digitales. Psicología, Conocimiento y Sociedad,6(1), 191-206. Recuperado de https://www.redalyc.org/jatsRepo/4758/475848615009/475848615009.pdf

Buzan, T. (1996): El libro de los mapas mentales. Editorial Urano. Barcelona, España.

Calderón, M. M. y Quesada, C. A. (2016). Los mapas mentales como estrategia didáctica para el mejoramiento de la comprensión lectora en textos narrativos. Recuperado de https://repository.unilibre.edu.co/handle/10901/8227

Campos, A. (2005): Mapas conceptuales, mapas mentales y otras formas de representación del conocimiento. Cooperativa Editorial Magisterio: Bogotá, Colombia.

Fernández, M. E., Vázquez, C. E. y López, M. E. (2016). Los mapas conceptuales multimedia en la educación universitaria: recursos para el aprendizaje significativo. Campus Virtuales, 5(1), 10-18. Recuperado de http://uajournals.com/ojs/index.php/campusvirtuales/article/view/100/99

Cordón, G. J. (2016). La investigación sobre lectura en el entorno digital. MEI: Métodos de información, 7(13), 247-268. Recuperado de http://dx.doi.org/10.5557/IIMEI7-N13-247268

Ugartetxea, G. J. (1996). La orientación metacognitiva. Un estudio sobre la capacidad transferencial de la metacognición y su influencia en el rendimiento intelectual. Revista de Psicodidáctica, (1), 27-53. Recuperado de https://www.redalyc.org/pdf/175/17517758004.pdf

Hernández, A. J. (2016) Mejoramiento de la comprensión de textos narrativos mediante la implementación del recurso educativo abierto Biblioteca Digital Ciudad Seva a través de la plataforma digital Edmodo (Tesis de Maestría). Recuperado de https://repository.javeriana.edu.co/bitstream/handle/10554/20434/HernandezAri asJorgeEnrique2016.pdf? sequence $=1$ yisAllowed $=\mathrm{y}$

Malhotra, N. (2008). Investigación de Mercados ( $5^{\mathrm{a}}$ ed.). Pearson Educación de México: Naucalpan de Juárez, México.

Martínez, M., Marrujo, J., Perillo, M., González, F. y Burin, D. (2019). Comprensión de texto en E-learning: estrategias de soporte y memoria de trabajo. Ocnos: Revista de estudios sobre lectura, 18(2), 31-43. Recuperado de http://dx.doi.org/10.18239/ocnos 2019.18.2.1988

Mendoza, C. S., García, S. L. Guzmán, O. M. (2018). El uso de Goconqr como herramienta que fortalece la formación profesional. Recuperado de http://www.conisen.mx/memorias2018/memorias/5/P814.pdf

Millalén, F. V. (2015). Impacto de la multimodalidad en la comprensión lectora de textos narrativos en inglés como lengua extranjera (L2) en estudiantes universitarios. Contextos educativos: Revista de educación, (18), 25-41. Recuperado de https://dialnet.unirioja.es/descarga/articulo/5080744.pdf

Mon, F. E. y Cervera, M. G. (2013). Competencia digital en la educación superior: instrumentos de evaluación y nuevos entornos.Enl@ce: Revista Venezolana de Información, Tecnología y Conocimiento, 10(3), 29-43. Recuperado de https://www.redalyc.org/pdf/823/82329477003.pdf

Novoa, C. P. (2019) El Mapa mental armónico en la comprensión y producción de textos narrativos en estudiantes de pregrado de la UCV (Tesis doctoral). Recuperado de http://repositorio.ucv.edu.pe/handle/UCV/35546

Novoa, C. P., Cancino Verde, Francisca, R., Flores Sotelo, W. S. y Nieto Gamboa, J. 
(2018). El Mapa Mental Armónico en la comprensión de textos narrativos en estudiantes universitarios. Propósitos y Representaciones, 6(2), 541-573. Recuperado de http://dx.doi.org/10.20511/pyr2018.v6n2.243.

Ortega, O. y Zózimo, R. (2012). Comprensión lectora, hábitos de estudio y rendimiento académico en estudiantes de primer año de una universidad privada de Lima Metropolitana (Tesis de Maestría). Recuperado de http://cybertesis.unmsm.edu.pe/handle/cybertesis/2738

Pérez-Rodríguez, A. y Ponce, Á. D. (2012). De la competencia digital y audiovisual a la competencia mediática: dimensiones e indicadores. Comunicar: Revista Científica de Comunicación y Educación, 20(39), 25-34. Recuperado de https://doi.org/10.3916/C39-2012-02-02

Piovano, S. y Burin, D. (2014). Comprensión y metacomprensión de los textos expositivos: Comparación experimental entre soporte impreso y el e-book reader. Recuperado de https://www.oei.es/historico/congreso2014/memoriactei/324.pdf

Ponce, H., López, M., Labra, J., Brugerolles, J. P. y Tirado, C. (2007). Evaluación experimental de un programa virtual de entrenamiento en lectura significativa (EPELS). Recuperado de http://repositorio.ual.es/bitstream/handle/10835/599/Art_12_169.pdf?sequence= $\underline{1}$

Pulgar, F. M. (2016). Incorporación de las herramientas tics para incrementar la comprensión lectora de textos narrativos en los estudiantes de primer ciclo de la facultad de derecho de una universidad privada de Lima. Recuperado de http://cort.as/-SpWs

Ramos, F. M. (2007). Nuevos avances en el estudio científico de la comprensión de textos. Universitas Psychologica, 6(2), 331-343. Recuperado de https://www.redalyc.org/pdf/647/64760212.pdf

Rodríguez, G. A. (2005). Lectura e internet: dos tecnologías. Investigación bibliotecológica, 19(38), 11-32. Recuperado de http://www.scielo.org.mx/pdf/ib/v19n38/v19n38a2.pdf

Rodríguez, R. A. y Vargas, A. C. H. (2017). Diferenciación de la interpretación de textos narrativos en la modalidad cuadernos digitales vs. ambientes tradicionales. Experiencias Investigativas y Significativas, 3(3). Recuperado de https://experiencias.iejuliussieber.edu.co/index.php/Exp-inv/article/view/23/13

Romero, L. (2014). Lectura tradicional versus lectura digital. Correspondencias y análisis, (4), 63-75. Recuperado de https://doi.org/10.24265/cian.2014.n4.03

Salazar, S. A., Sevilla, R.O., González, P. B., Mendoza, A. C., Echeverri, G. A., Quecán, C. D., Pardo, R.L, Angulo, A. M., Silva, G. J. y Lozano, R. M. (2015). Lectura y escritura en la universidad: contribución para reconstruir una historia. Magis. Revista Internacional de Investigación en Educación, 8(16), 51-70. Recuperado de https://doi.org/10.11144/Javeriana.m8-16.leuc

Saldarriaga, M. L. (2017). Fortalecimiento de la comprensión inferencial a través de textos narrativos con contenido social (Tesis de licenciatura). Recuperado de http://hdl.handle.net/20.500.12209/7732

Thorne, C., Morla, K., Uccelli, P., Nakano, T., Mauchi, B., Landeo, L., Vásquez, A. y Huerta, R. (2013). Efecto de una plataforma virtual en comprensión de lectura y vocabulario: Una alternativa para mejorar las capacidades lectoras en primaria. Revista de Psicología (PUCP), 31(1), 3-35. Recuperado de 


\section{http://www.scielo.org.pe/pdf/psico/v31n1/a01v31n1.pdf}

Farrach, U. G. (2016). Estrategias metodológicas para fomentar la comprensión lectora. Revista Científica de FAREM-Estelí, (20), 5-19. Recuperado de https://doi.org/10.5377/farem.v0i20.3064

Villalustre, M. L. y Del Moral, P. M. (2010). Mapas conceptuales, mapas mentales y líneas temporales: objetos "de" aprendizaje y "para" el aprendizaje en Ruralnet. Revista Latinoamericana de Tecnología Educativa-RELATEC, 9(1), 15-27. Recuperado de https://relatec.unex.es/article/view/602/434

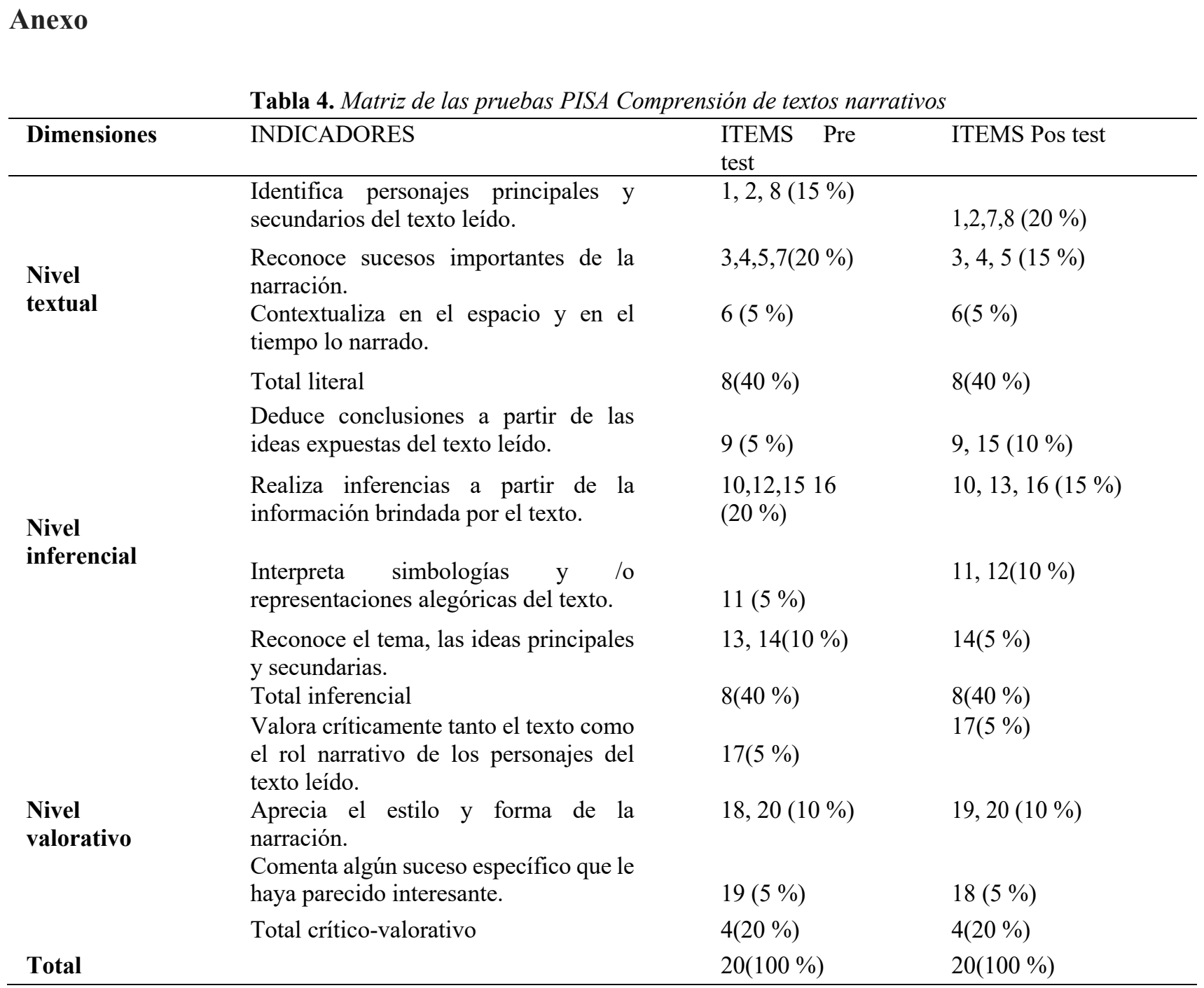




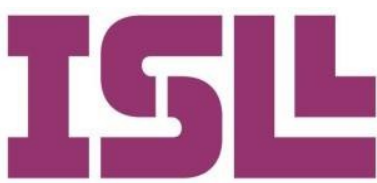

Number 11

2019 June

\title{
Digital application strategies in understanding narrative texts
}

\author{
Pedro Félix Novoa Castillo \\ Universidad Nacional Mayor de San Marcos/ \\ Universidad César Vallejo \\ https://orcid.org/0000-0003-2186-7458
}

Received: 03-03-2019

Accepted: 25-04-2019

\section{Abstract:}

The poor reading comprehension in the university sector requires a necessary alternative solution from the didactic. Therefore, digital application strategies are an interesting solution proposal. The objective of this research was to determine the influence of a set of applications and programs used as a teaching strategy in the process of reading and understanding narrative texts. To verify this, a pre and two post test (PISA adaptations, 2015) was applied to a sample of 310 : control (160) and experimental (150). The results reflected significant differences in understanding, in the results of the evaluation of entry and exit according to study groups. Therefore, it was concluded that Digital Application Strategies significantly improve the understanding of narrative texts. 


\section{Introduction}

In this research we sought to verify the influence of a digital strategy in the understanding of narrative texts by university students. Because of this, we focused on proposing activities that would have to have, in one or two classes at most, some intense examination of the relevance of virtual resources, programs and applications for achieving literal, inferential, and criteria understanding. The readings used were two stories by Jorge Luis Borges: "The garden of the paths that bifurcate" and "The secret miracle."

Research on text comprehension is lush and yet the results remain tentative and preliminary regarding an effective solution to where there is little or no reading comprehension. Unfortunately, there is a marked bias to not take narrative texts into account, underestimating their flexibility, and avoiding or stereotyping their relevance. Those who approach them reduce the narrative text to mere entertainment without analysis or verification of understanding. And those who study prefer to use argumentative or expository texts, assuming superior rigor, wasting the narrative enclave in current communications, as they are the basis of these times in communication. And also, not taking advantage of narrative texts that are short of extension are complex as the works of Borges.

Finally, when choosing a quasi-experimental design, the present research proposes a cross-section of the research, assuming that only in theses (and very little in articles) are the approaches of the experiments that seek to influence the understanding of the texts observed. There is also a bit of specific research approach with university students in relation to narrative texts. For all this, this work assumes to contribute with results that motivate people to continue fighting for a society that understands what it reads, so to also understand what it is living.

\section{Reading and Understanding in Digital Environments}

Reading in a physical or in digital environment creates a great pedagogical dilemma in its use in both media. The physical texts are concrete and fixed to the environment that facilitates it; while digital is intangible and virtual (Cordón, 2016). These differences disturbed the intellectuals who thought they could become precarious not only the reading itself, but their understanding. However, Piovano \& Burin (2014) have found no differences in understanding. In spite of this, users and specialists have noted that there is a great qualitative difference between reading in physical and in a digital environment when it comes to bulky texts. In fact, in this type of reading the monotonous light of the screens causes a visual fatigue in the reader. It can even cause "dry eye" because of its excessive exposure. Also, if the reading is given on mobile devices, this reduces the holistic vision of an entire page as in a specific book reading. Even so, the studies were decanting these disadvantages as simple peculiarities, understanding that each of them had their own characteristics and therefore their intrinsic advantages and disadvantages.

For some pro-digital researchers, virtual reading on electronic devices meant more 
interaction and dynamism than reading on simple pages. But that was not why they lacked disruptive elements. In order to avoid cognitive overload, the digital reader had to have specific skills to take advantage of all the advantages of virtual text and reduce its disadvantages (Cordón, 2016). In this same sense, Romero (2014) states that it is necessary for readers to be effective readers who relate to the writing in a more fluid, effective, and constant way. Skills such as design, search, and navigation (Fajardo et al.; $\mathrm{Wu}, 2014$ cited in Burin et al., 2016). This is why this research has highlighted the design of activities that take into account the interactive and previous knowledge (Martínez et al., 2019) as well as the search for cognitive conflict to round understanding.

\section{Understanding Narrative Texts}

Understanding a text is understood as a mental exercise that involves effort and the approach of an intellectual purpose (Ramos, 2007), along with a kind of interaction between reader and text in actively building a certain intellection (Blandón \& Restrepo, 2015). If the text to be understood is of the narrative type, the succession of events, the situation and setting of each character involved in a story will be taken into account (Calderón \& Quesada, 2016) along a time sequence (Saldarriaga, 2017). The narrative is the type of holistic text par excellence, since within it are other types of text, both today and since the beginning of time (Barthes, 1996).

It is important to underline that within the most read texts we have novels and stories. The first, the novels, are the most voluminous and are therefore related to a physical support par excellence. This is unlike the latter, which can be accessed more easily both physically, but especially digitally, because of their short length. However, they are not being used as they should, since, according to Salazar et al. (2015), only $6 \%$ of texts is established for reviewing short stories as short stories.

Short narrative texts are usually used as an induction to more complex and farreaching later readings. They have been used in the transition from the reader from Bisoño to the most used reader (Acevedo et al., 2013; Arciniega \& Chiriboga, 2015). However, they should not be reduced to considering narratives as providing simple links from the simple to the complex or from the short to the extensive, since they can be short and complex in themselves. In addition, they are, in these times, the most used communication vehicles, especially on digital platforms.

This is why this study has used the stories "The Secret Miracle" and "The Garden of Paths that Fork." They are two short stories by Borges, who is considered to be a writer who is characterized by his conciseness and complexity.

\section{Level of Impact of Digital Programs and Applications}

To work both reading and subsequent understanding, a didactic strategy was chosen that took into account the levels of impact of technology in education. According to Puentedura (2006) and López (2015) as cited in Mendoza et al. (2018), this impact can include: (1) improving and transforming with a moderate substitution of the physical for the digital; (2) increasing the real in the virtual with a direct and functional substitution; 
(3) modifying the real in the virtual with a direct, functional substitution and the use of specific didactic strategies; and (4) modification of the real in the virtual with a substitution and the use of ICT tools in the creation of new learning.

In this investigation, the third level of impact was chosen. This was because both the programs and applications used sought a direct replacement of physical reading with a virtual one in a functional way, as well as the use of specific didactic strategies to perform specific pedagogical processes such as collecting prior knowledge, cognitive conflict, recovery and organization of information, and evaluation of reading comprehension.

The criteria for choosing the applications and programs were the ones that were most intuitive and did not imply cognitive overload or require much difficulty for their access or sophistication in their processing; since, in some occasions, the ICT tools instead of being a means, are involuntarily transformed into an end in themselves (Levis, 2016 cited in Pérez-Rodríguez \& Ponce, 2012). This was in addition to having, in most cases, economic conditions for their subscriptions.

Therefore, this research used the following programs and applications: Seva, Kahoot, miMind, Educaplay, Goconqr, and Thatquiz digital library. The Seva Digital Library was used to access the two stories used in this work. Kahoot was used for the collection of previous knowledge and cognitive conflict. miMind was ideal for retrieving and organizing information in Harmonic Mind Maps. The Educaplay platform was very important for the design and application of post-reading recreational activities such as word search, crossword puzzles, and interactive maps. Finally, both Goconqr and Thatquiz served to evaluate the readings.

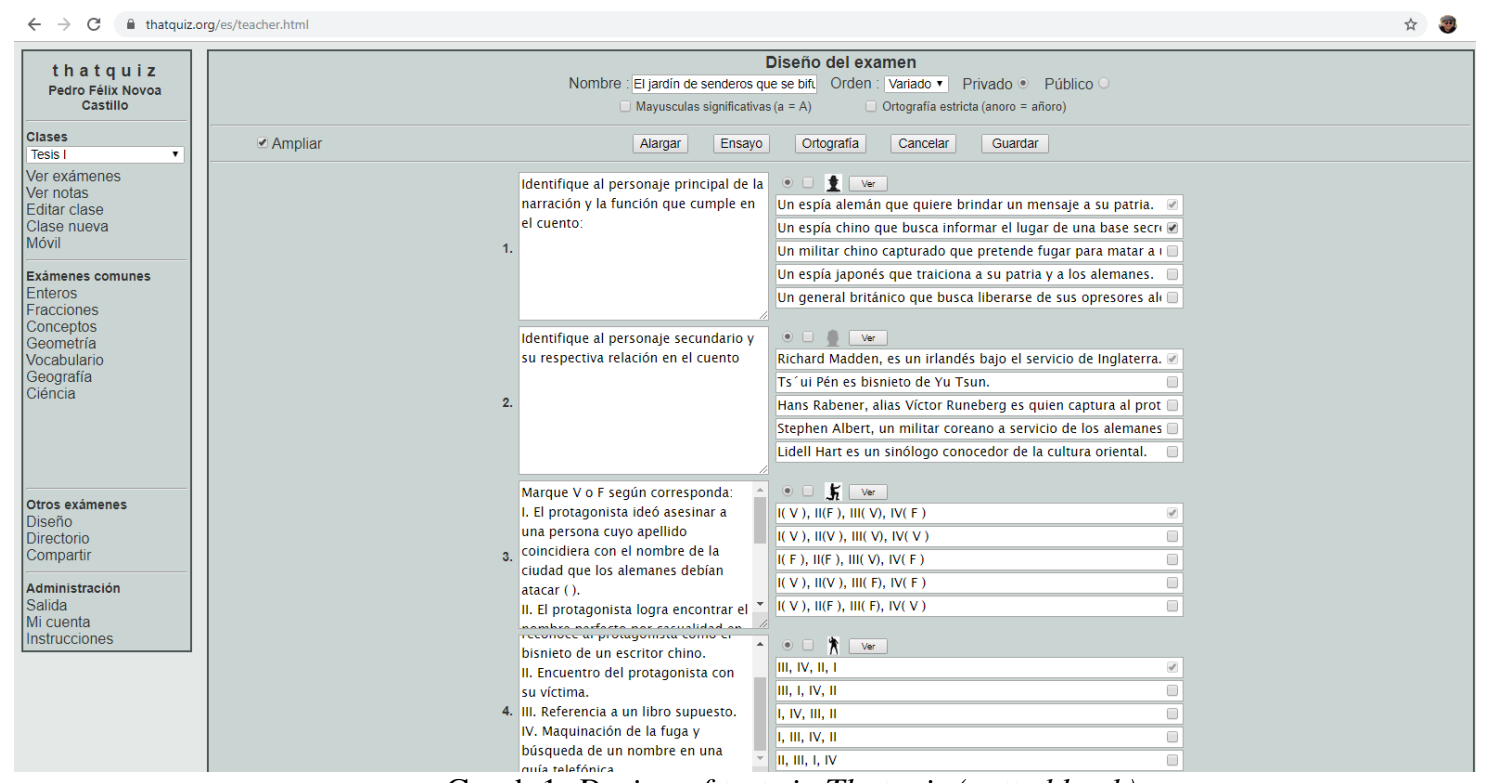

Graph 1. Design of tests in Thatquiz (cutted back)

It is necessary to note that the use of technology in education is still low. Salazar et al. (2015) revealed that only $6 \%$ of schools used electronic devices for educational purposes in Colombia. It should be added that, due to poverty, the technological gap in Latin America is greater (Bravo, 2012; Rodríguez, 2005) that other countries and 
precisely because of this, the requirement for teachers to make the most of the available resources. It is in this context that strategies in digital environments become the ideal means to positively influence learning.

After ensuring learning, the immediate thing is evaluation. For this, this work opted for online tests. Modality, according to Thorne et al. (2013) and Ponce et al. (2007) is an alternative that, if well used, can improve results. In this investigation, a pretest and two posttests (one previous and one definitive) were applied, with the understanding that carrying out an additional evaluation as an essay offers additional security of improving the total understanding of the text read (Benítez et al., 2014).

Online assessments, in turn, have the advantage of being immediate, fast, and with the option of self-regulation according to each learning pace. The researchers recommend that reading assessments appeal to a contextualized design (Mon \& Cervera, 2013) and that each question about reading seeks a deep and meaningful understanding (Martínez et al. 2019).

\section{Harmonic Mind Maps in Digital}

An essential resource for the strategy used in this research was the use of Harmonic Mind Maps. These are understood to be improved version of the Buzan classics (1996), since a negative characteristic is eliminated as the motley of the original, and it is replaced by the harmonic, making the branches lose their disorder and gain a balanced and more harmonious distribution (Novoa, 2019; Novoa et al., 2018), maintaining their characteristics in a more relevant way, as the new maps use figures or icons with a high semantic load (Fernández et al., 2016).

With these information organizers it was possible to retrieve, sort, and rank the newly acquired information in the reading. For design the strategy we chose that digital resource, since these present benefits, such as the reticular or bifurcated organization in real time, creative and free (Apollo et al., 2016).

This organizer is within the cognitive approach that lately has a lot of adherence in the educational community (Salazar et al., 2015). This is because within the studies related to learning, the Mind Maps allow for the construction of new knowledge based on their management, organization, and hierarchy, involving relationships and senses that link them, creating a kind of mental image of a specific content (Campos, 2005; Villalustre \& Del Moral, 2010). Likewise, the Mind Maps are considered didactically as flexible and effective in the task of consolidating learning (Ortega \& Zósimo, 2012), since these visual organizers allow to organize specific segments of knowledge in an interrelated way.

To perform these schemes, the miMind platform was used in this investigation. 


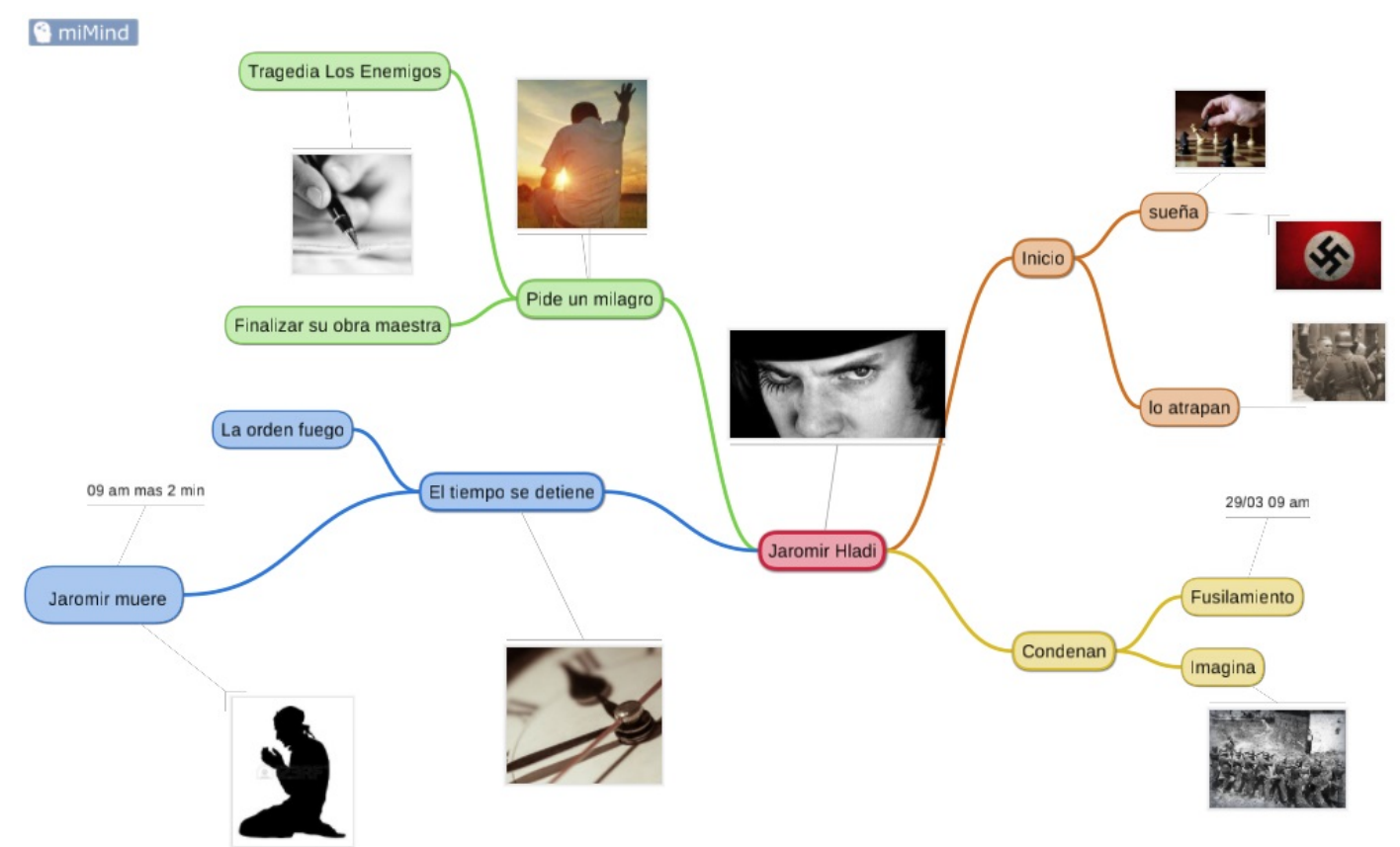

Graph 2. Organization of what is read in a Harmonic Mind Map in digital (miMind)

\section{Objectives and Hypotheses}

The research aimed to demonstrate that certain programs and applications of a practical and simple nature, used as a digital strategy by teachers, positively influence the reading comprehension of narrative texts (general objective), including in its textual, inferential, and criterial dimensions (specific objective).

Due to the hypothetical deductive method of the present work, the following hypotheses were raised:

1. The digital strategy improves the understanding of narrative texts (general hypothesis).

2. The digital strategy improves the textual, inferential, and criterial understanding of narrative texts (specific hypothesis).

\section{Method}

Type of study: We worked with an experimental research design of the quasiexperimental type of cross-section, due to the need to verify the effects of a strategy based on the use of certain digital programs and applications and considering that the experience should be cutting cross. Also, with this methodological design you can also experience the strategy to measure its impact.

\section{Participants}

The research focused on a population of 1580 students of the Faculty of Education of the UNMSM, of which a sample of 310 participants was taken (Control Group: 160 and Experimental Group: 150) of oscillating ages between 18 to 27 years, through a simple probabilistic sampling (Malhotra, 2008). Those selected signed an informed consent document and were given their respective return of results. 


\section{Instruments/Materials}

Two Borges narratives available online (Ciudad Seva) were used: "The Garden of the Paths That Fork" as a pretest reading and "The Secret Miracle" as a posttest reading. Both texts are of similar complexity, although they have different word counts (3747 and 2242). Therefore, a reading time of 37 minutes was assigned for the first story and 22 minutes for the second.

Three PISA-type tests were applied for the comprehension of narrative texts (Based on Novoa et al., 2018; Novoa, 2019) where, through multiple alternative questions, information on literal, inferential, and criterial understanding was collected: a pretest in the Thatquiz platform, a first posttest in the Goconqr environment and a last posttest in Thatquiz.

A short two-minute questionnaire was used in Kahoot, before reading; and after reading a Digital Mind Map through the miMind platform, for 15 minutes; Three recreational activities were carried out through the Educaplay platform: a crossword puzzle (10 minutes), a word search (10 minutes), and an interactive map (5 minutes).

\section{Procedure}

In the first class, the signature of the informed consent is made, the text "The Garden of the Bifurcated Paths" is read and afterward, the pretest is applied to both the Control Group and the Experimental Group.

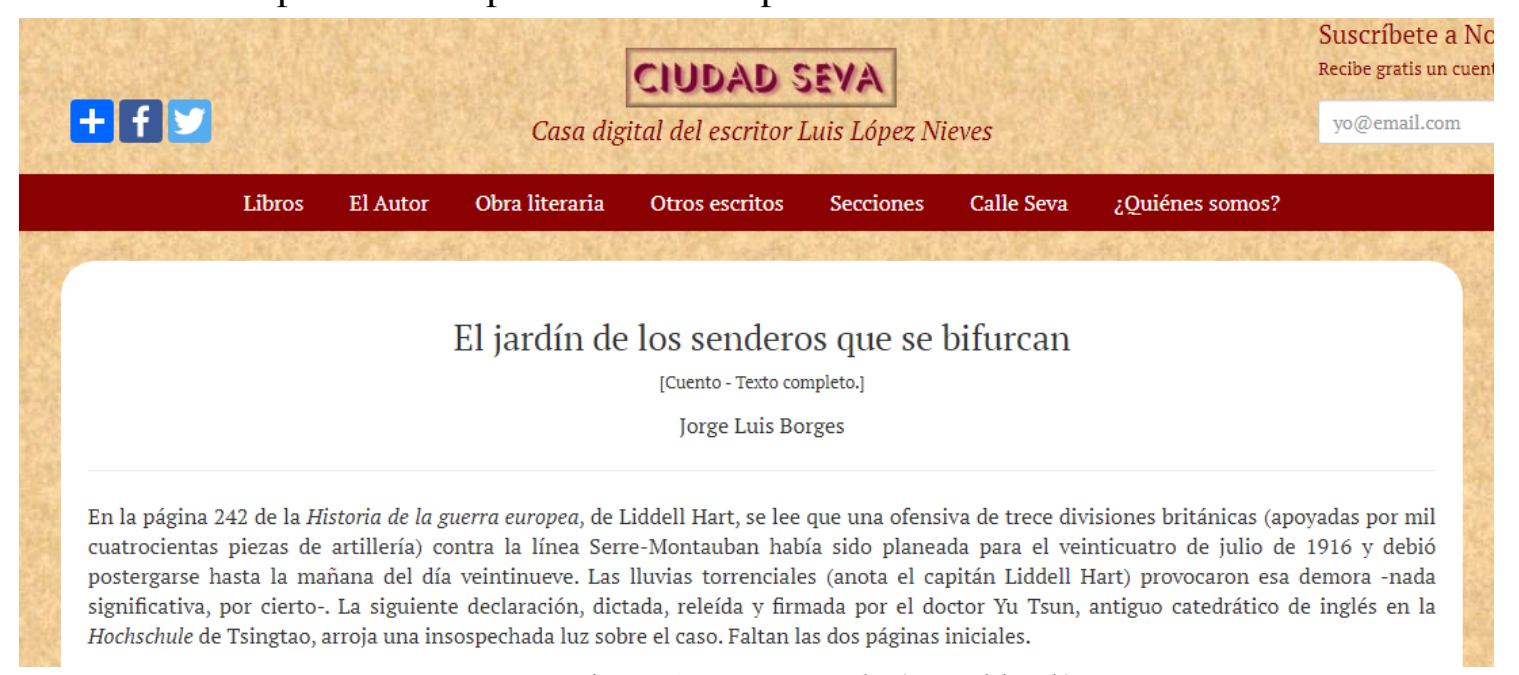

Figure 1. Pre test Tale (cutted back) 


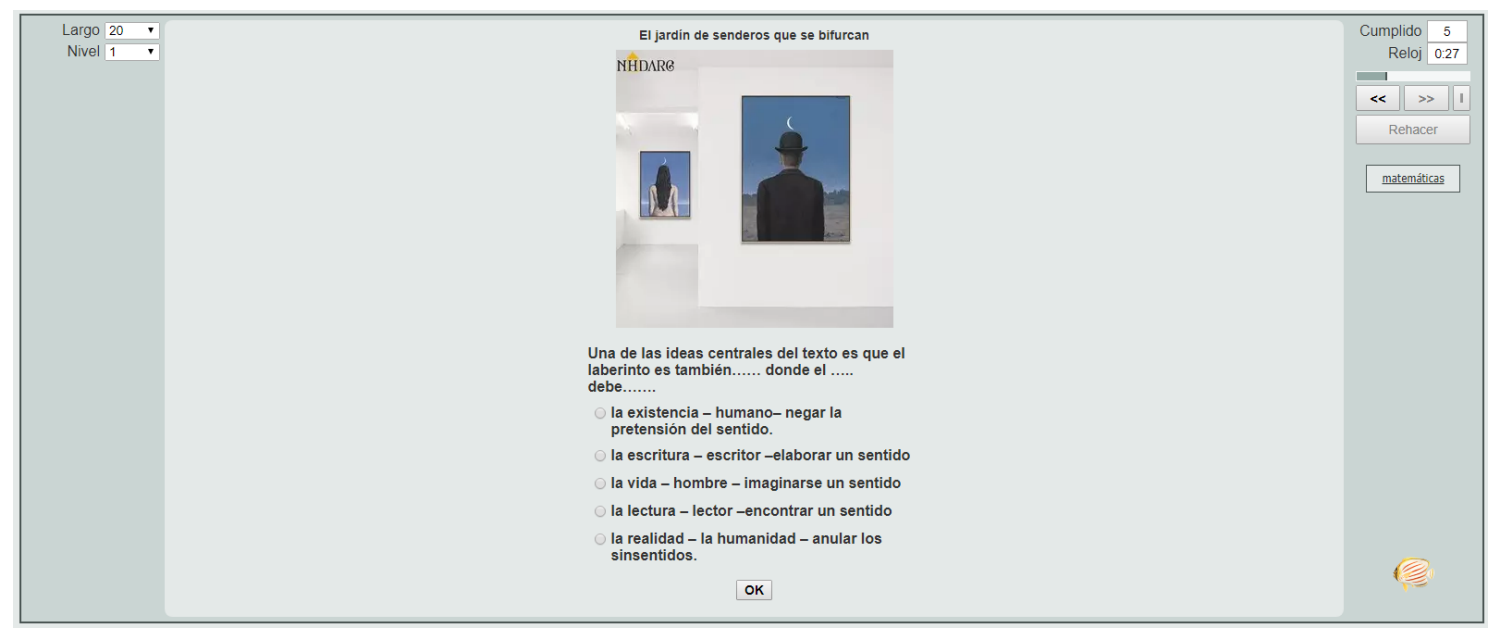

Figure 2. Pre test (cutted back)

In the second class, a recovery of the previous knowledge is developed with the Experimental Group and cognitive conflict is sought through a brief Kahoot questionnaire, where the first two questions are for the collection of previous knowledge, which is important to anchor and relate to new information; and the last two to provoke a cognitive conflict from reflection on time, crucial theme of the story to be read. The conflict arises from the statement that man is or is not mired in time. The statement is true, but it is used to cause an imbalance in the acquisition of new learning (Campione et al., 1987; Piaget, 1985; Siegler \& Richards, 1989 as cited in Ugartetxea, 1996).

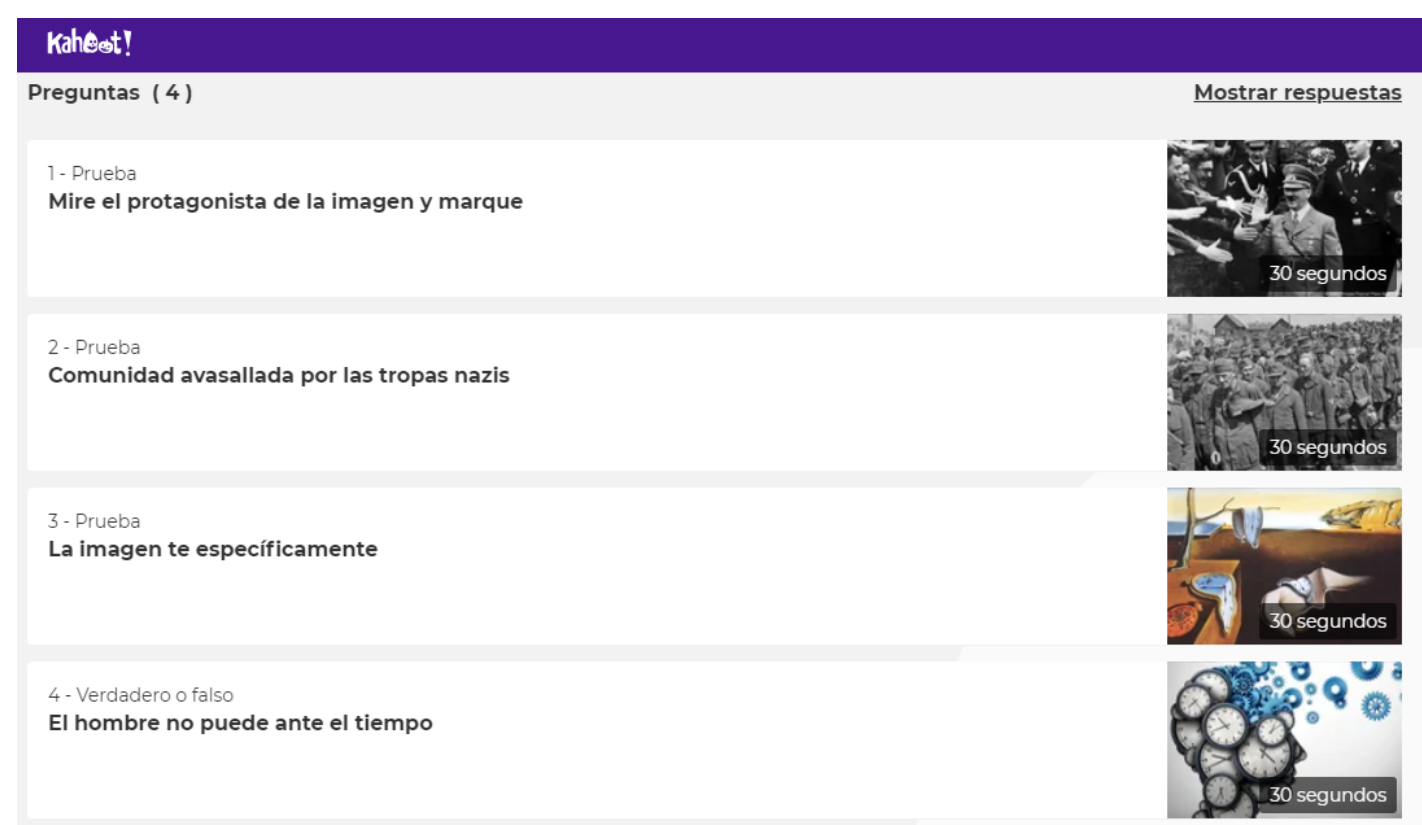

Figure 3. Brief Kahoot questionnaire (cutted back)

After the dynamics of Kahoot, we proceed to read "The Secret Miracle." After this, the first posttest is carried out. Some reflections on the questions that have raised doubts in the students are socialized. An information organization is made through the development of a Harmonic Mind Map on the miMind platform. It continues with three recreational activities that will serve as reinforcers of new knowledge: a crossword puzzle, to refer to related topics; a soup of letters, to reinforce keywords; and an 
interactive map that will ensure the narrative sequence of the story read. Incorporating playfulness into strategies to encourage reading comprehension is important, since it allows a reduction of the complexity and difficulty of the comprehension process (Farrach, 2016).

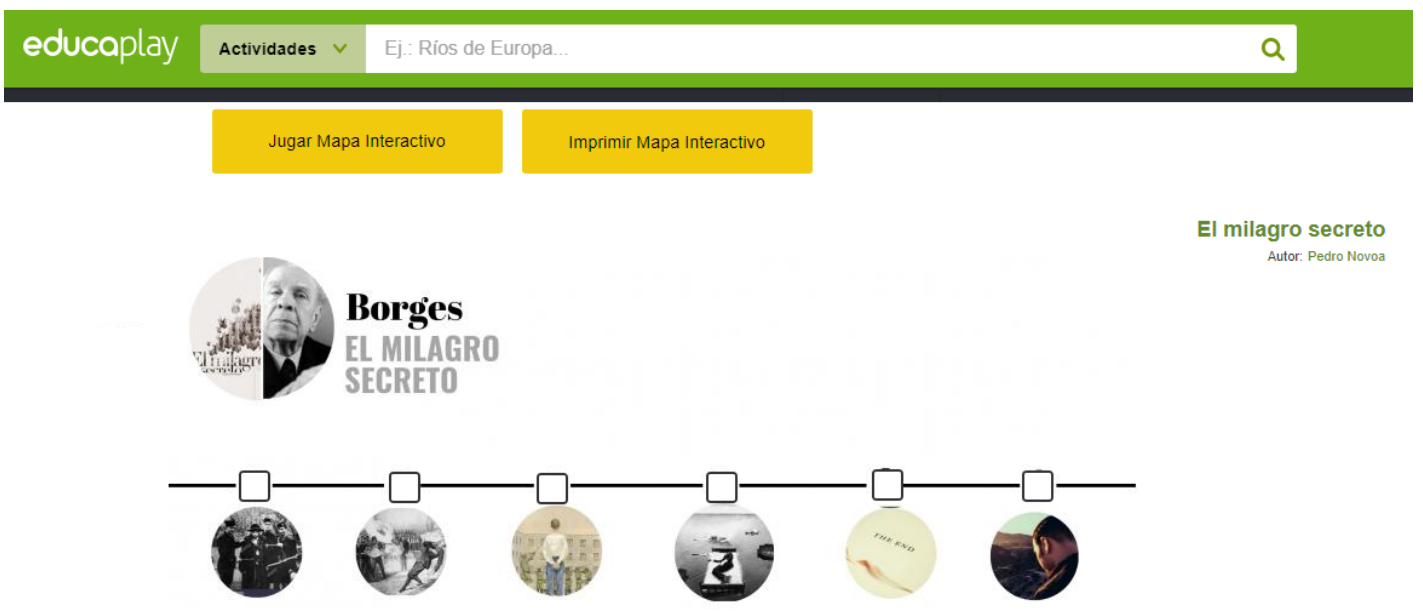

Figure 4. Educaplay interactive map (cutted back)

Finally the last post test is taken.

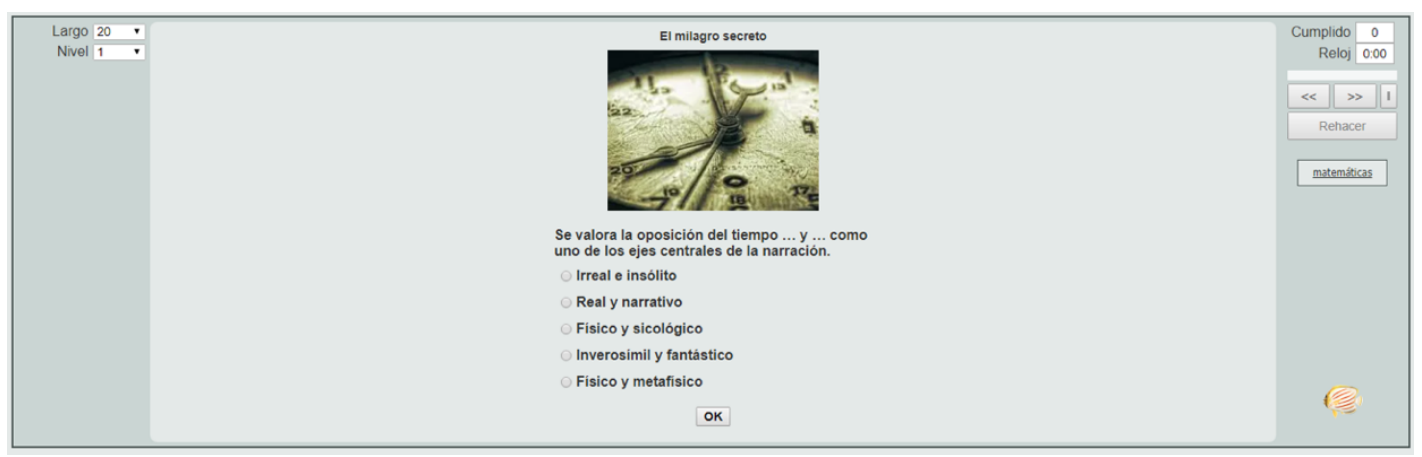

Figure 5. Thatquiz last post test (cutted back)

\section{Results}

The narrative text comprehension notes were analyzed and the normality test was applied.

Table 1. Normality test of comprehension of narrative text

\begin{tabular}{ccccc}
\hline Kolmogorov-Smirnov $^{\mathbf{a}}$ & test & Statistics & gl & Sig. \\
\hline Narrative texts & pre control & .153 & 160 & .000 \\
comprehension & pre experimental & .175 & 150 & .000 \\
& pos control & .140 & 160 & .000 \\
& pos experimental & .174 & 150 & .000 \\
& a. Lilliefors significance correction
\end{tabular}

And since the $\mathrm{p}$ value of significance was less than .005 , it was determined that 
the data presented a non-normal distribution. Because of this, the Mann-Whitney U test was established as a non-parametric test for subsequent hypothesis testing.

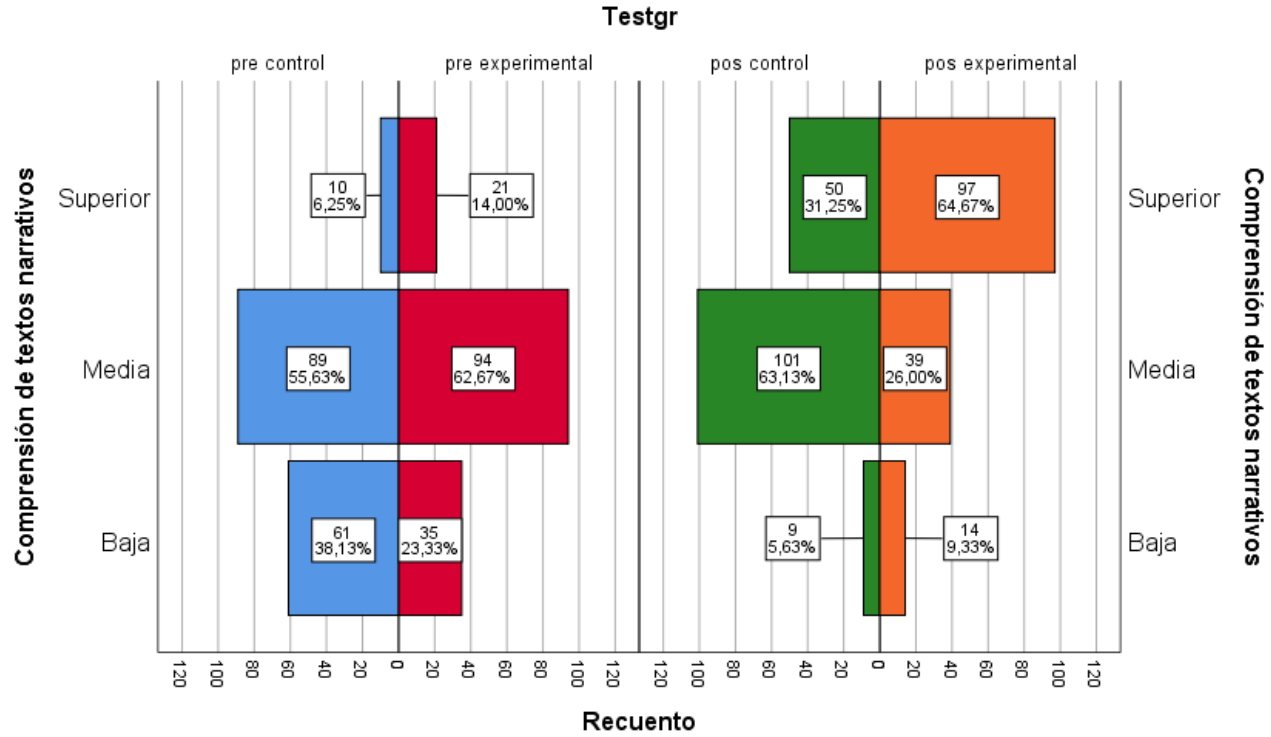

Graph 3. Result of the Comprehension Tests of narrative texts

It is observed in Graph 3 that in the pretest of comprehension of narrative texts both the Control Group and the Experimental Group were in similar conditions, although with small differences. In contrast, in the posttest, these differences were highlighted in favor of the Experimental Group, since a significant percentage of the low level rose to a medium level and another large percentage rose from the middle to the upper level.

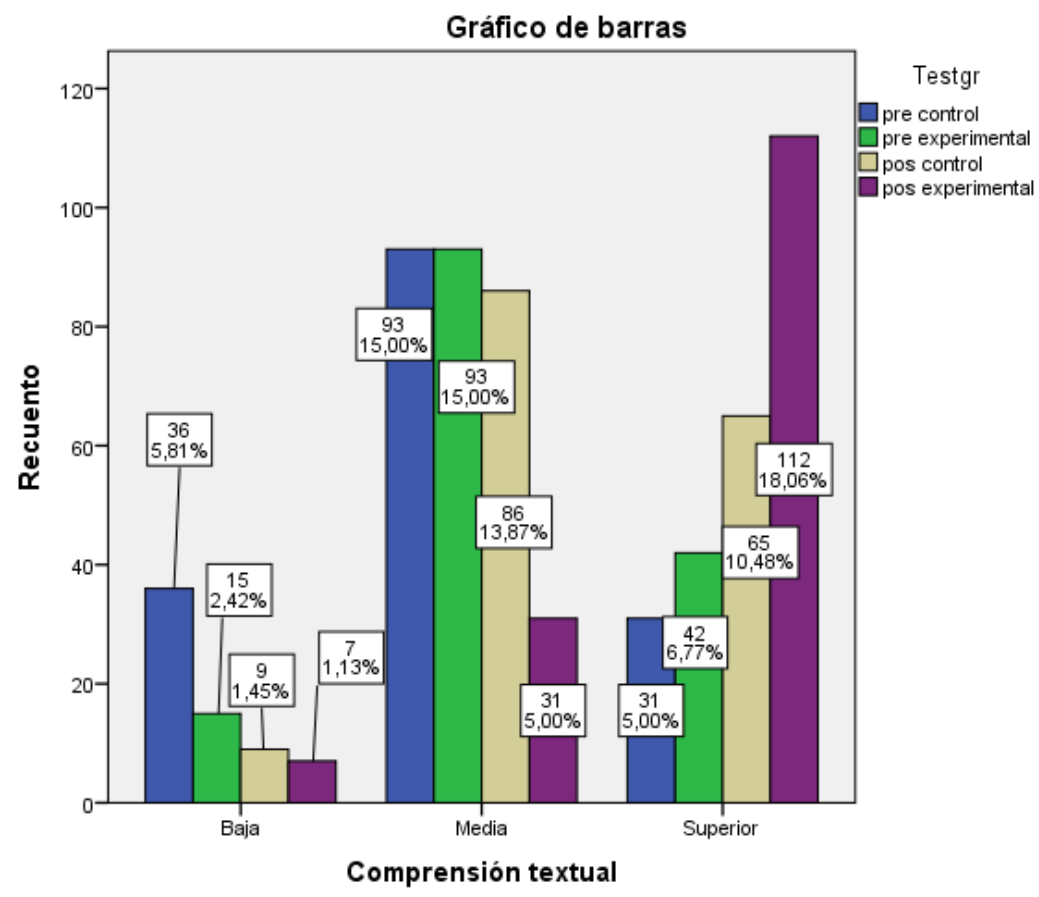

Graph 4. Textual comprehension of narrative texts by evidence

Figure 4 shows that the Textual Understanding dimension in the pretest shows slight differences in the lower and upper levels, while in the posttest, there is a marked difference in favor of the Experimental Group. 


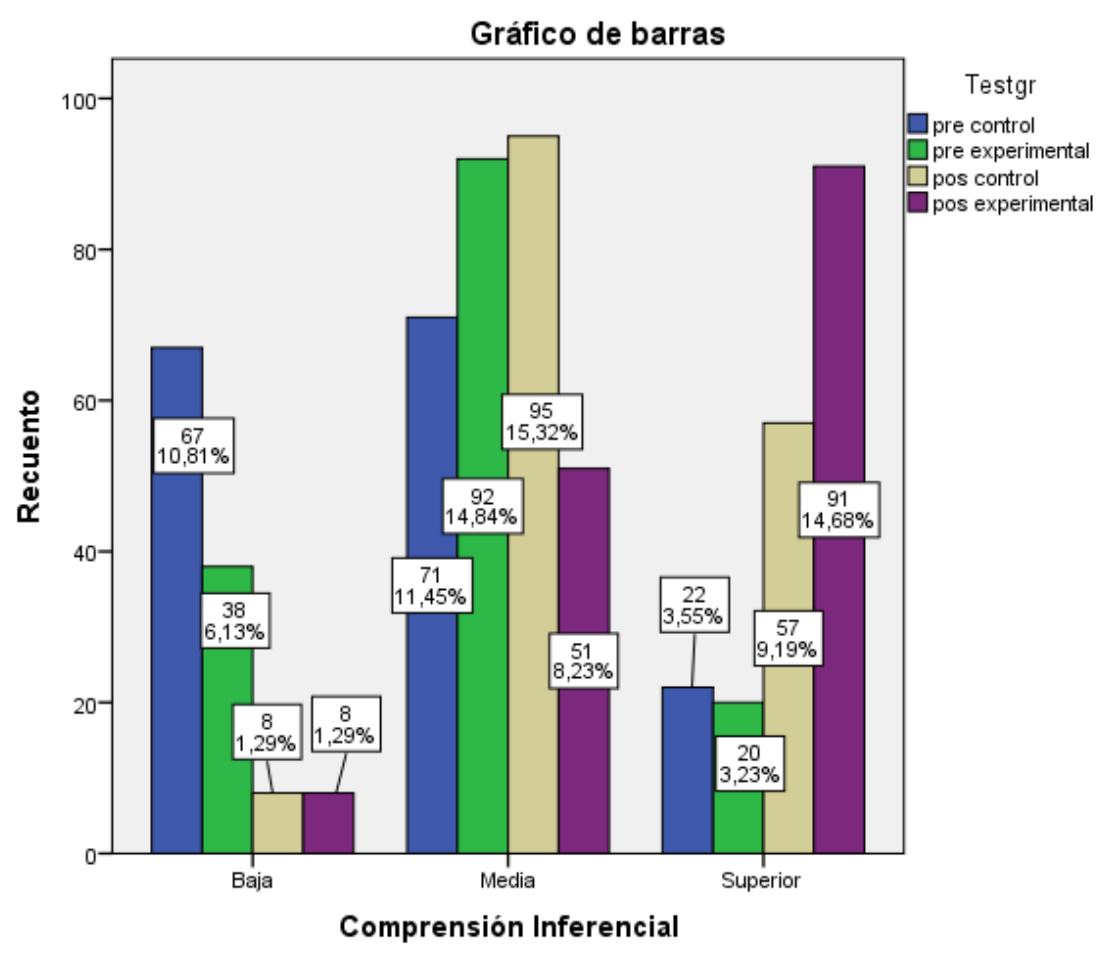

Graph 5. Inferential understanding of narrative texts by evidence

Graph 5 shows that the inferential understanding dimension in the pretest shows slight differences in the low, medium, and higher levels, while in the posttest, there is a marked difference in favor of the Experimental Group at the upper level, but none at the low level; there is a rather great advantage of the Control Group at the medium level.

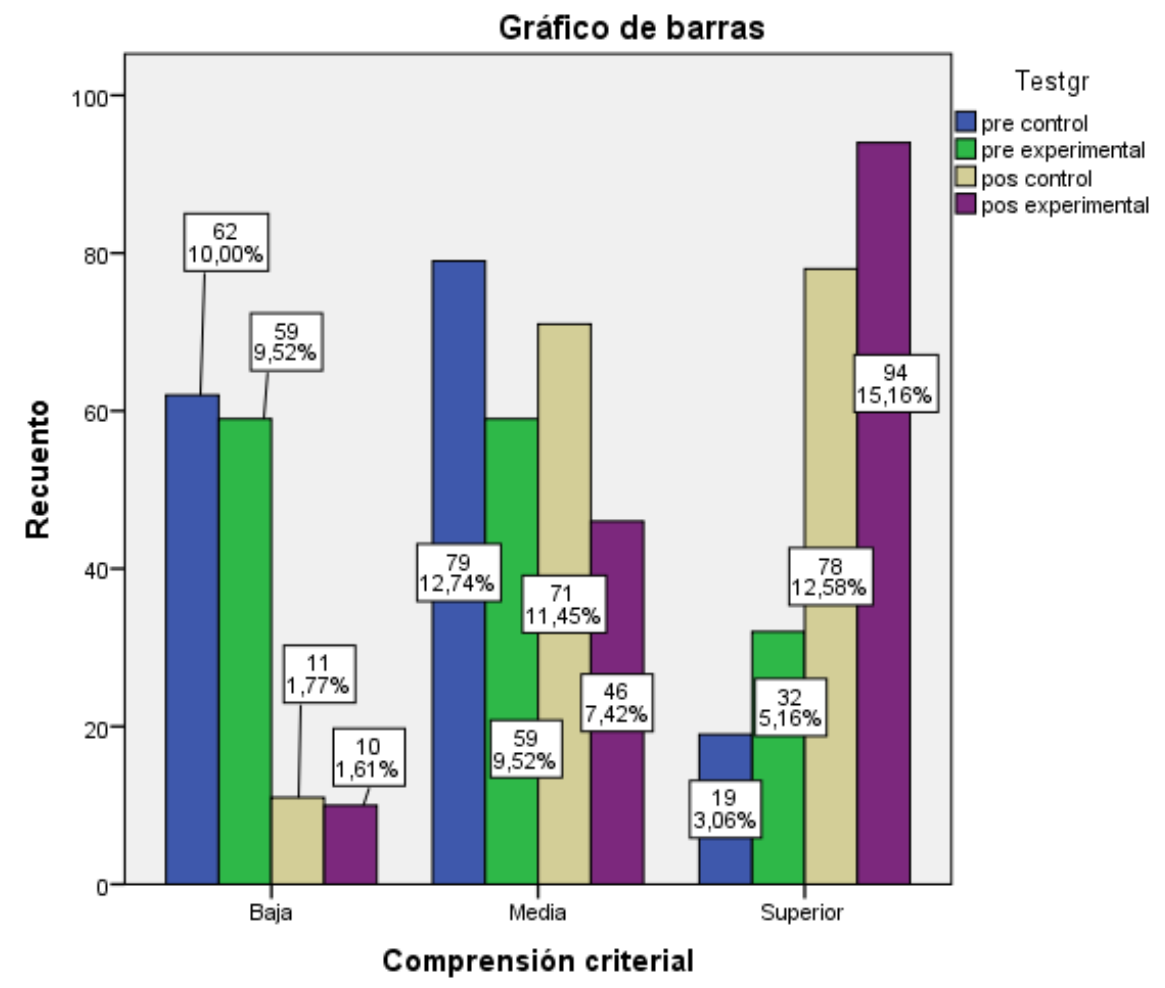

Graph 6. Criterial comprehension of narrative texts by evidence

Graph 6 shows that the criterion comprehension dimension in the pretest shows slight differences in the low, medium, and higher levels, while in the posttest, there is a 
marked difference in favor of the Experimental Group at the upper level; but not in the middle, where the Control Group has a predominance.

\section{Inferential results}

General hypothesis test:

$\mathrm{H}_{0}$ : Digital application strategies do not improve the understanding of narrative texts.

$\mathrm{H}_{\mathrm{i}}$ : Digital application strategies improve understanding of narrative texts.

Tabla 2. Prueba U de Mann-Whitney para probar la hipótesis general

\begin{tabular}{|c|c|c|c|c|c|c|}
\hline \multicolumn{5}{|c|}{ Ranges } & \multicolumn{2}{|c|}{ Contrast statistics } \\
\hline \multirow{6}{*}{$\begin{array}{l}\text { Narrative } \\
\text { texts } \\
\text { comprehen } \\
\text { sion }\end{array}$} & Test y Grupo & $N$ & $\begin{array}{l}\text { Rango } \\
\text { prom. }\end{array}$ & $\begin{array}{l}\text { Suma } \\
\text { rangos }\end{array}$ & & \\
\hline & Pre Control & 160 & 162.69 & 26030.00 & $\begin{array}{c}\text { Ude Mann-Whitney } \\
\text { W de Wilcoxon }\end{array}$ & $\begin{array}{l}10850.000 \\
22175.000\end{array}$ \\
\hline & Pre Experimental & 150 & 147.83 & 22175.00 & $Z$ & -1.479 \\
\hline & & & & & $\begin{array}{l}\text { Sig; } p \text {. Asintót. } \\
\text { (bilateral) }\end{array}$ & .139 \\
\hline & Pos Control & 160 & 134.88 & 21580.00 & $\begin{array}{c}\text { Ude Mann-Whitney } \\
W \text { de Wilcoxon }\end{array}$ & $\begin{array}{l}8700.000 \\
21580.000\end{array}$ \\
\hline & Pos Experimental & 150 & 177.50 & 26625.00 & $\begin{array}{c}Z \\
\begin{array}{c}\text { Sig; p. Asintót. } \\
\text { (bilateral) }\end{array}\end{array}$ & $\begin{array}{l}-4.216 \\
.000\end{array}$ \\
\hline
\end{tabular}

It is observed in Table 2 that in the pretest of comprehension of narrative texts, the ranges of the Control Group and the Experimental Group are not so differentiated. In addition, both the $\mathrm{p}$ value of significance (greater than $\alpha=.05$ ) and the $\mathrm{Z}$ value (greater than the critical limit -1.96) confirm that there are no significant differences between the two groups. However, in the posttest, there is a marked difference in the ranges. In addition, it is appreciated that $\mathrm{p}$. of significance (greater than $\alpha=.05$ ) and the $\mathrm{Z}$ value (less than the critical limit -1.96) confirm that there are significant differences between both groups. Therefore, the null hypothesis is rejected and it is concluded that Digital application strategies significantly improve the comprehension of narrative texts.

Specific hypothesis test

$\mathrm{H}_{0}$ : Digital application strategies do not improve textual comprehension, inferential understanding, and criterial comprehension of narrative texts.

$\mathrm{H}_{\mathrm{i}}$ : Digital application strategies do not improve text comprehension, inferential understanding, and criterial comprehension of narrative texts.

It is observed in Table 3 that in the pretest of textual comprehension, inferential comprehension, and criterial comprehension of narrative texts, the ranks of the Control Group and the Experimental Group are not so differentiated. In addition, both the p value of significance (greater than $\alpha=.05$ ) and the $\mathrm{Z}$ value (greater than the critical limit -1.96 ) confirm that there are no significant differences between the two groups. However, in the posttest, there is a marked difference in the ranges of textual comprehension and criterial 
comprehension of narrative texts. In addition, it is appreciated that the $\mathrm{p}$ of significance (less than $\alpha=.05$ ) and the $Z$ value (less than the critical limit -1.96) corroborate that there are significant differences between both groups. This is except for inferential understanding, which maintained both its similar ranks and that both its $p$ value of significance and value $\mathrm{Z}$ also remained high, so the influence in this dimension is not significant. For all these reasons, the null hypothesis is partially rejected and it is concluded that Digital application strategies significantly improve textual comprehension and criterial comprehension of narrative texts.

\section{Discussion}

In the present investigation, some activities were tested in digital environments with the objective that students read and understand short narrative texts. The application of this type of strategy has been of great impact in the understanding of narrative texts. These results enter into a dialogic controversy with what was found by Pulgar (2016), who also used a quasi-experimental design to corroborate the significance of the incorporation of ICT tools as a strategy to improve the understanding of narrative texts in university students. He concluded that the effect was $76.9 \%$ in general reading comprehension at the end of his quasi-experimental investigation. The incidence in literal comprehension was from 12.8 to $56 \% ; 7.7$ to $66 \%$ in inferential understanding; and 23.1 to $41 \%$ in criterial understanding. These results are significantly higher than those found in the present work; however, that would be signified by a certain bias in the data processing when having a normal distribution and therefore the use of non-parametric tests such as the Student's T-test. Also, it is observed that in the program used in Pulgar's study there are texts that are not narrative, but expository; and that in turn the level of difficulty of the readings is dissimilar in their complexity (the author chooses very short texts or fragments, while in this investigation Borges' complete stories were chosen).

Another immediate reference is the research carried out by Novoa et al. (2018), who find a significant influence using the physical version of the digital variant used in this work: the harmonic mental map. In this research a significance is achieved $(\mathrm{p}=.000)$, checking the working hypothesis, that the Harmonic Mind Maps significantly influence the understanding of narrative texts.

The importance of adding digital elements to the pedagogical strategies, such as in the case of Millalén (2015), who carried out a quasi-experimental investigation to verify the effects of multimodality in the understanding of narrative texts in university students comes into convergence with what was found in this research. The author showed that the Experimental Group scored 55.6 and the Control Group 38.8 in general understanding. This advantage was verified by the Student's T-test $(\mathrm{t}=6.381)$ and a significance of $\mathrm{p}=.000$, accepting with this our working hypothesis as in this investigation. However, it should be noted that in Millalén's study, the text was in English and oriented to a second language.

In this same context of taking advantage of ICT environments, Hernández (2016) used a mixed approach to verify that the comprehension of narrative texts improves through a digital library and a virtual platform (Edmodo). The author proved that there was a relevant improvement in the syntactic, semantic, and pragmatic levels. In this case, 
the present investigation also coincides in the positive influence, but not in the instruments used, on in the dimensions, since the present work opted for the canonical instruments, which are literal, inferential, and criterial understanding.

With regard to reading and understanding in digital environments, the present work found better results in the Experimental Group, which had a permanent activity in digital environments. These findings complement the results of Rodríguez \& Vargas (2017), who found slight advantages in the interpretation of narrative texts of the participants who used digital notebooks with those who used traditional environments.

In conclusion, this research offers the possibility of carrying out a set of combined strategies both before and after reading a narrative text and improving the results of understanding. These strategies are usually intuitive, flexible, and especially active, and they constitute an aspect not yet studied in depth in relation to the understanding of narrative texts mediated by digital strategies in the university environment.

\section{Conclusions}

It was found that the use of a digital strategy based on the use of virtual programs and applications in a transversal way (one or two days) has a significant effect on the understanding of narrative texts.

It was evidenced that there was improvement in the dimensions of textual comprehension and criterial comprehension, but that the significance in the inferential understanding was not achieved, most likely due to the fact that there is a rush in the deductions of the participants, a very common situation in youth of these times.

It was demonstrated that the use of digital programs and applications that are intuitive to use and of little complexity and which have low interference in educational activities have good results both in their applicability and in the results that show improvement.

\section{References}

Acevedo, V. M., Cabana P., \& Martínez V. L (2013). Estrategias pedagógicas para el desarrollo de procesos lectores y escriturales orientados a la articulación entre el nivel preescolar y la básica primaria(Thesis). Recovered from http://hdl.handle.net/11227/5364

Arciniega T. R., \& Chiriboga L. J. (2015). Comprensión lectora de fábulas para la formación de valores enfocados en el buen vivir en los alumnos del cuarto grado de las escuelas Mercedes Troya de Suárez y Víctor Manuel Peñaherrera de la ciudad de Ibarra en el año lectivo 2012; 2013. Propuesta alternativa (Tesis de Bachiller). Recuperado de http://repositorio.utn.edu.ec/handle/123456789/4008

Apolo, D., Bayés, M., \& Hermann, A. (2016). Cambios educativos en los procesos de lectura digital: la pedagogía del ciberespacio como estrategia de procesamiento de contenidos en la era de internet. Revista de Estudios para el Desarrollo Social de la Comunicación, (12), 222 en http://dx.doi.org/10.15213/redes.n12.p222

Barthes, R. (1966). Introducción al análisis estructural del relato. Buenos Aires. Tiempo 
Contemporáneo.

Benítez, L., Barajas, V., Uresti, H., \& Nallehly, I. (2014). Efecto de la aplicación de una estrategia de comprensión de lectura en un entorno virtual. Revista electrónica de investigación educativa, 16(3), 71-87. Recovered from http://www.scielo.org.mx/scielo.php?pid=S1607$40412014000300005 y$ script $=$ sci arttextytlng=en

Blandón, M. V., \& Restrepo, L. F. (2015). La comprensión de textos narrativos: implementación de una secuencia didáctica de enfoque comunicativo, con estudiantes de grado segundo(Tesis doctoral). Recovered from https://core.ac.uk/download/pdf/71399434.pdf

Bravo, E. (2012). Globalización, innovación tecnológica y pobreza. Aproximación a las nuevas conceptualizaciones en Latinoamérica. Espacio abierto, 21(3), 543-556. Recuperado de https://www.redalyc.org/pdf/122/12223296007.pdf

Burin, D., Coccimiglio, Y., González, F., \& Bulla, J. (2016). Desarrollos recientes sobre habilidades digitales y comprensión lectora en entornos digitales. Psicología, Conocimiento y Sociedad,6(1), 191-206. Recovered from https://www.redalyc.org/jatsRepo/4758/475848615009/475848615009.pdf

Buzan, T. (1996). El libro de los mapas mentales. Barcelona: Editorial Urano.

Calderón, M. M., \& Quesada, C. A. (2016). Los mapas mentales como estrategia didáctica para el mejoramiento de la comprensión lectora en textos narrativos. Recovered from https://repository.unilibre.edu.co/handle/10901/8227

Campos, A. (2005): Mapas conceptuales, mapas mentales y otras formas de representación del conocimiento. Cooperativa Editorial Magisterio. Bogotá.

Cordón, G. J. (2016). La investigación sobre lectura en el entorno digital. MEI: Métodos de información, 7(13), 247-268 en http://dx.doi.org/10.5557/IIMEI7-N13$\underline{247268}$

Farrach, U. G. (2016). Estrategias metodológicas para fomentar la comprensión lectora. Revista Cientifica de FAREM-Esteli, 20, 5-19 Recovered from https://doi.org/10.5377/farem.v0i20.3064

Fernández, M. E., Vázquez, C. E., \& López, M. E. (2016). Los mapas conceptuales multimedia en la educación universitaria: recursos para el aprendizaje significativo. Campus Virtuales, 5(1), 10-18. Recovered from http://uajournals.com/ojs/index.php/campusvirtuales/article/view/100/99

Hernández, A. J. (2016) Mejoramiento de la comprensión de textos narrativos mediante la implementación del recurso educativo abierto Biblioteca Digital Ciudad Seva a través de la plataforma digital Edmodo (Tesis de Maestría). Recovered from https://repository.javeriana.edu.co/bitstream/handle/10554/20434/HernandezAri asJorgeEnrique2016.pdf? sequence $=1$ yisAllowed $=\mathrm{y}$

Malhotra, N. (2008). Investigación de Mercados ( $5^{\mathrm{a}}$ ed.). Naucalpan de Juárez, México: Pearson Educación de México.

Martínez, M., Marrujo, J., Perillo, M., González, F., \& Burin, D. (2019). Comprensión de texto en E-learning: estrategias de soporte y memoria de trabajo. Ocnos: Revista de estudios sobre lectura, 18(2), 31-43 in http://dx.doi.org/10.18239/ocnos 2019.18.2.1988

Mendoza, C. S., García, S. L., \& Guzmán, O. M. (2018). El uso de Goconqr como herramienta que fortalece la formación profesional. Recovered from 
http://www.conisen.mx/memorias2018/memorias/5/P814.pdf

Millalén, F. V. (2015). Impacto de la multimodalidad en la comprensión lectora de textos narrativos en inglés como lengua extranjera (L2) en estudiantes universitarios. Contextos educativos: Revista de educación, 18, 25-41. Recuperado de https://dialnet.unirioja.es/descarga/articulo/5080744.pdf

Mon, F. E., \& Cervera, M. G. (2013). Competencia digital en la educación superior: instrumentos de evaluación y nuevos entornos.Enl@ce: Revista Venezolana de Información, Tecnología y Conocimiento, 10(3), 29-43. Recovered from https://www.redalyc.org/pdf/823/82329477003.pdf

Novoa, C. P. (2019) El Mapa mental armónico en la comprensión y producción de textos narrativos en estudiantes de pregrado de la UCV (Thesis). Recovered from http://repositorio.ucv.edu.pe/handle/UCV/35546

Novoa, C. P., Cancino, F., R., Flores, W. S., \& Nieto, J. (2018). El Mapa Mental Armónico en la comprensión de textos narrativos en estudiantes universitarios. Propósitos y Representaciones, 6(2), 541-573 in http://dx.doi.org/10.20511/pyr2018.v6n2.243.

Ortega, O., \& Zózimo, R. (2012). Comprensión lectora, hábitos de estudio y rendimiento académico en estudiantes de primer año de una universidad privada de Lima Metropolitana (Thesis of master). Recovered from http://cybertesis.unmsm.edu.pe/handle/cybertesis/2738

Pérez-Rodríguez, A., \& Ponce, Á. D. (2012). De la competencia digital y audiovisual a la competencia mediática: dimensiones e indicadores. Comunicar: Revista Científica de Comunicación y Educación, 20(39), 25-34. Recovered from https://doi.org/10.3916/C39-2012-02-02

Piovano, S., \& Burin, D. (2014). Comprensión y metacomprensión de los textos expositivos: Comparación experimental entre soporte impreso y el e-book reader. Recovered from https://www.oei.es/historico/congreso2014/memoriactei/324.pdf

Ponce, H., López, M., Labra, J., Brugerolles, J. P., \& Tirado, C. (2007). Evaluación experimental de un programa virtual de entrenamiento en lectura significativa (E-PELS). Recovered from http://repositorio.ual.es/bitstream/handle/10835/599/Art_12 169.pdf?sequence $=1$

Pulgar, F. M. (2016). Incorporación de las herramientas tics para incrementar la comprensión lectora de textos narrativos en los estudiantes de primer ciclo de la facultad de derecho de una universidad privada de Lima. Recovered from http://cort.as/-SpWs

Ramos, F. M. (2007). Nuevos avances en el estudio científico de la comprensión de textos. Universitas Psychologica, 6(2), 331-343. Recovered from https://www.redalyc.org/pdf/647/64760212.pdf

Rodríguez, G. A. (2005). Lectura e internet: dos tecnologías. Investigación bibliotecológica, 19(38), 11-32. $\quad$ Recovered from http://www.scielo.org.mx/pdf/ib/v19n38/v19n38a2.pdf

Rodríguez, R. A., \& Vargas, A. C. H. (2017). Diferenciación de la interpretación de textos narrativos en la modalidad cuadernos digitales vs. ambientes tradicionales. Experiencias Investigativas y Significativas, 3(3). Recovered from 
https://experiencias.iejuliussieber.edu.co/index.php/Exp-inv/article/view/23/13 Romero, L. (2014). Lectura tradicional versus lectura digital. Correspondencias y análisis, (4), 63-75 en https://doi.org/10.24265/cian.2014.n4.03

Salazar, S. A., Sevilla, R.O., González, P. B., Mendoza, A. C., Echeverri, G. A., Quecán, C. D., Pardo, R.L, Angulo, A. M., Silva, G. J., \& Lozano, R. M. (2015). Lectura y escritura en la universidad: contribución para reconstruir una historia. Magis. Revista Internacional de Investigación en Educación, 8(16), 51-70 en https://doi.org/10.11144/Javeriana.m8-16.leuc

Saldarriaga, M. L. (2017). Fortalecimiento de la comprensión inferencial a través de textos narrativos con contenido social (Tesis de licenciatura). Recovered from http://hdl.handle.net/20.500.12209/7732

Thorne, C., Morla, K., Uccelli, P., Nakano, T., Mauchi, B., Landeo, L., Vásquez, A., \& Huerta, R. (2013). Efecto de una plataforma virtual en comprensión de lectura y vocabulario: Una alternativa para mejorar las capacidades lectoras en primaria. Revista de Psicología (PUCP), 31(1), 3-35. Recovered from http://www.scielo.org.pe/pdf/psico/v31n1/a01v31n1.pdf

Ugartetxea, G. J. (1996). La orientación metacognitiva. Un estudio sobre la capacidad transferencial de la metacognición y su influencia en el rendimiento intelectual. Revista de Psicodidáctica, (1), 27-53. Recovered from https://www.redalyc.org/pdf/175/17517758004.pdf

Villalustre, M. L., \& Del Moral, P. M. (2010). Mapas conceptuales, mapas mentales y líneas temporales: objetos "de" aprendizaje y "para" el aprendizaje en Ruralnet. Revista Latinoamericana de Tecnología Educativa-RELATEC, 9(1), 15-27. Recovered from https://relatec.unex.es/article/view/602/434 


\section{Annexed}

Table 4. Matrix of the PISA tests Understanding narrative texts

\begin{tabular}{|c|c|c|c|}
\hline Dimensions & INDICATORS & ITEMS Pre test & ITEMS Pos test \\
\hline \multirow{3}{*}{$\begin{array}{l}\text { Textual } \\
\text { level }\end{array}$} & $\begin{array}{l}\text { Identify main and secondary characters of the } \\
\text { read text. }\end{array}$ & $1,2,8(15 \%)$ & $1,2,7,8(20 \%)$ \\
\hline & Recognize important events in the story. & $3,4,5,7(20 \%)$ & $3,4,5(15 \%)$ \\
\hline & $\begin{array}{l}\text { Contextualized in the space and in the time it } \\
\text { narrated. }\end{array}$ & $6(5 \%)$ & $6(5 \%)$ \\
\hline \multirow{6}{*}{$\begin{array}{l}\text { Inferential } \\
\text { level }\end{array}$} & Literal total & $8(40 \%)$ & $8(40 \%)$ \\
\hline & $\begin{array}{l}\text { Deduces conclusions on from the } \\
\text { ideas exposed in the text read. }\end{array}$ & $9(5 \%)$ & $9,15(10 \%)$ \\
\hline & $\begin{array}{l}\text { Makes inferences to from the information pro } \\
\text { vided by the text. }\end{array}$ & $\begin{array}{l}10,12,15,16 \\
(20 \%)\end{array}$ & $\begin{array}{l}10,13,16(15 \\
\%)\end{array}$ \\
\hline & $\begin{array}{l}\text { Interpret symbologies and / } \\
\text { or allegorical representations of the text. }\end{array}$ & $11(5 \%)$ & $11,12(10 \%)$ \\
\hline & $\begin{array}{l}\text { Recognize the topic, } \\
\text { the main and secondary ideas. }\end{array}$ & $13,14(10 \%)$ & $14(5 \%)$ \\
\hline & Inferential total & $8(40 \%)$ & $8(40 \%)$ \\
\hline \multirow{4}{*}{$\begin{array}{l}\text { Level } \\
\text { invidious }\end{array}$} & $\begin{array}{l}\text { Rate critically both } \\
\text { the text and the role narrative of } \\
\text { the characters of the text read. }\end{array}$ & $17(5 \%)$ & $17(5 \%)$ \\
\hline & Appreciate the style and form of the story. & $18,20(10 \%)$ & $19,20(10 \%)$ \\
\hline & $\begin{array}{l}\text { Discuss any event specific that has seemed in } \\
\text { teresting. }\end{array}$ & $19(5 \%)$ & $18(5 \%)$ \\
\hline & Critical-Value Total & $4(20 \%)$ & $4(20 \%)$ \\
\hline Total & & $20(100 \%)$ & $20(100 \%)$ \\
\hline
\end{tabular}

